\title{
Orientation-Selective Adaptation to First- and Second-Order Patterns in Human Visual Cortex
}

\author{
Jonas Larsson, Michael S. Landy, and David J. Heeger \\ Department of Psychology and Center for Neural Science, New York University, New York, New York
}

Submitted 27 June 2005; accepted in final form 4 October 2005

Larsson, Jonas, Michael S. Landy, and David J. Heeger. Orientation-selective adaptation to first- and second-order patterns in human visual cortex. J Neurophysiol 95: 862-881, 2006. First published October 12, 2005; doi:10.1152/jn.00668.2005. Second-order textures-patterns that cannot be detected by mechanisms sensitive only to luminance changes - are ubiquitous in visual scenes, but the neuronal mechanisms mediating perception of such stimuli are not well understood. We used an adaptation protocol to measure neural activity in the human brain selective for the orientation of second-order textures. Functional MRI (fMRI) responses were measured in three subjects to presentations of first- and second-order probe gratings after adapting to a high-contrast first- or second-order grating that was either parallel or orthogonal to the probe gratings. First-order (LM) stimuli were generated by modulating the stimulus luminance. Second-order stimuli were generated by modulating the contrast $(\mathrm{CM})$ or orientation $(\mathrm{OM})$ of a first-order carrier. We used four combinations of adapter and probe stimuli: LM:LM, CM:CM, OM:OM, and LM: OM. The fourth condition tested for cross-modal adaptation with first-order adapter and second-order probe stimuli. Attention was diverted from the stimulus by a demanding task at fixation. Both firstand second-order stimuli elicited orientation-selective adaptation in multiple cortical visual areas, including V1, V2, V3, V3A/B, a newly identified visual area anterior to dorsal V3 that we have termed LO1, $\mathrm{hV} 4$, and VO1. For first-order stimuli (condition LM:LM), the adaptation was no larger in extrastriate areas than in V1, implying that the orientation-selective first-order (luminance) adaptation originated in V1. For second-order stimuli (conditions CM:CM and OM:OM), the magnitude of adaptation, relative to the absolute response magnitude, was significantly larger in VO1 (and for condition CM:CM, also in $\mathrm{V} 3 \mathrm{~A} / \mathrm{B}$ and LO1) than in V1, suggesting that second-order stimulus orientation was extracted by additional processing after V1. There was little difference in the amplitude of adaptation between the secondorder conditions. No consistent effect of adaptation was found in the cross-modal condition LM:OM, in agreement with psychophysical evidence for weak interactions between first- and second-order stimuli and computational models of separate mechanisms for first- and second-order visual processing.

\section{N T R O D U C T I O N}

Much of the early visual system from the retina to the primary visual cortex (V1) is devoted to detecting luminance changes. To a first approximation, the underlying neural mechanisms can be described in terms of linear filters applied to the retinal image. The receptive field of a V1 simple cell, for instance, can be approximated by a Gabor function having a particular location, orientation, spatial frequency, and spatial phase, and the responses of such a cell to an arbitrary visual stimulus can be predicted by summing the luminance intensity

Address for reprint requests and other correspondence: J. Larsson, Dept. of Psychology, New York Univ., 6 Washington Place, New York, NY 10003 (E-mail: jonas@cns.nyu.edu). over all points within the receptive field, weighted by the value of the Gabor function at each point, followed by thresholding the result (corresponding to the neuronal spiking threshold). However, there are many types of image structure that cannot be detected by a linear filter. Consider the photograph of a cobblestone road in Fig. 1A. The old and new sections of paving in the road differ by the size and pattern of the stonework, i.e., their texture, but the average luminance intensity remains roughly constant across the sections. A linear filter tuned to luminance variations would therefore be unable to distinguish the two road sections. Patterns that cannot be detected by linear filters are often referred to as second-order patterns to distinguish them from luminance-defined, firstorder patterns (those visible to a linear mechanism). Another type of second-order stimulus is a pattern varying in contrast (Fig. 1B). A linear mechanism (indicated by the superimposed cartoon receptive field) tuned to the spatial frequency and orientation of a first-order stimulus with the same spatial modulation frequency and orientation (Fig. 1C) would yield a net zero response to this second-order stimulus. Other types of second-order patterns can be generated by varying the local orientation, spatial frequency, or phase of an underlying finescale pattern (referred to as the carrier stimulus).

Second-order pattern perception is characterized by several unique properties indicating that first- and second-order patterns are detected and represented by different neuronal processing mechanisms. For instance, modulation contrast sensitivity for second-order patterns, unlike that for first-order stimuli, does not depend strongly on the spatial frequency of the second-order modulating pattern (Landy and Oruç 2002). Rather, the visibility of second-order patterns is determined (among other factors) by the ratio between carrier and modulator spatial frequencies (Sutter et al. 1995). The optimal ratio is constant over a large range of spatial scales, implying that second-order vision is scale invariant (Dakin and Mareschal 2000; Kingdom et al. 1995; Sutter et al. 1995). Like their first-order counterparts, second-order mechanisms are tuned for orientation and spatial frequency (Arsenault et al. 1999; Sutter et al. 1995), but have greater bandwidth (Landy and Oruç 2002). The strongest evidence for separate mechanisms come from psychophysical measurements of interactions between first- and second-order stimuli (Ellemberg et al. 2004; Ledgeway and Smith 1994; Morgan et al. 2000; Schofield and Georgeson 1999; Scott-Samuel and Georgeson 1999). These studies have found either weak or incomplete interaction be-

\footnotetext{
The costs of publication of this article were defrayed in part by the payment of page charges. The article must therefore be hereby marked "advertisement" in accordance with 18 U.S.C. Section 1734 solely to indicate this fact.
} 

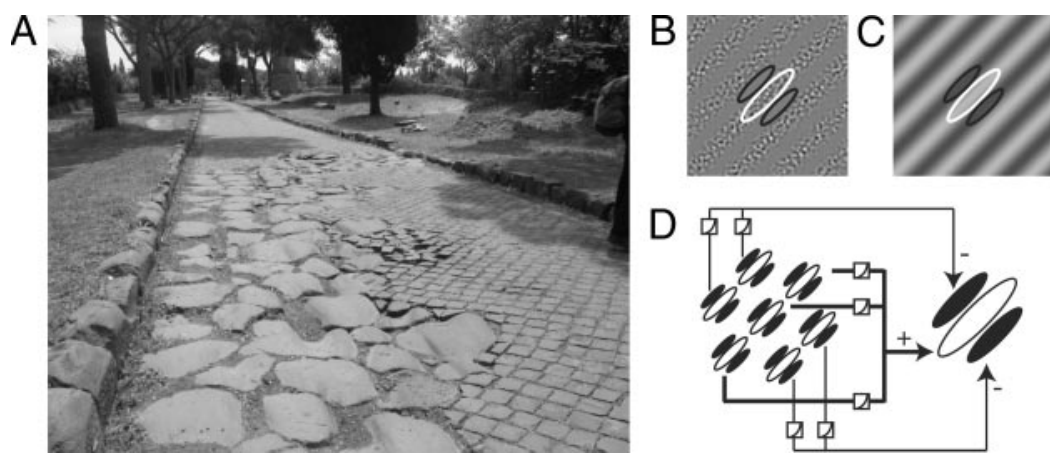

FIG. 1. A: example of naturally occurring 2nd-order patterns. Texture variations not associated with luminance changes in this image (a photograph of Via Appia) cannot be detected by a 1st-order, linear filter measuring only brightness variations, but are visible to a 2 nd-order filter that measures variations in spatial frequency. $B$ : pure 2 nd-order patterns are invisible to 1 st-order filters. A 1st-order Gabor-type linear filter (indicated by the black and white ellipses, corresponding to inhibitory and excitatory lobes) yields a net 0 response to a contrast modulated grating. $C$ : same filter superimposed on a 1st-order (luminance modulated) grating having the same spatial frequency and orientation. Net luminance differs between excitatory and inhibitory lobes, yielding a strong filter response. $D$ : filter-rectify-filter (FRF) model of 2nd-order visual processing. Outputs of many 1st-order filters (indicated by small Gabor patterns to the left) are rectified and summed by a 2 nd-order filter tuned to a lower spatial frequency.

tween the two stimulus categories, implying that they are not processed by a single mechanism.

Models of second-order visual processing postulate the existence of a three-stage mechanism variously referred to as the filter-rectify-filter (FRF), linear-nonlinear-linear (LNL), or "back pocket" model (see Landy and Graham 2004 for a review). In this model, the outputs of linear units tuned to the spatial frequency of the carrier stimulus are rectified and pooled by a larger linear filter tuned to the orientation and spatial frequency of the second-order modulation (Fig. 1D). FRF models provide a relatively simple mechanism to account for second-order pattern perception that could be implemented in neuronal circuitry. The properties of the first-stage filters in these models are similar to those of simple cells in V1. The outputs of several such neurons are assumed to be pooled by neurons corresponding to the second-stage filter, the rectification stage being implemented by the spiking threshold of the first-order neurons.

Neurons with properties consistent with the second-stage filter have been found in cat and monkey visual cortex. Singleunit studies have identified neurons selective for a variety of second-order stimuli, such as patterns defined by modulations of carrier orientation (Olavarria et al. 1992; Rossi et al. 2001), contrast (Leventhal et al. 1998; Mareschal and Baker 1998a,b, 1999; O'Keefe and Movshon 1998; Zhou and Baker 1993, 1994, 1996), spatial frequency (Leventhal et al. 1998), temporal frequency (Albright 1992; Chaudhuri and Albright 1997), and phase shifts of abutting gratings (a type of illusory contour) (Grosof et al. 1993; von der Heydt and Peterhans 1989; von der Heydt et al. 1984). Most of these neurons were found in extrastriate visual areas (area 18 in the cat, V2 and MT in macaque monkey), although a small number of neurons selective for second-order patterns were found in V1. Interestingly, most neurons selective for second-order patterns also responded to first-order patterns having similar properties to the second-order modulating stimulus (e.g., orientation and spatial frequency), suggesting that such neurons encode a cue-invariant representation of the stimuli. Cue-invariant neurons responding to shapes and gratings defined by texture, luminance, motion, or color, have also been found in inferotemporal cortex of monkeys (Sary et al. 1993, 1995). Although cue-invariance for different types of second-order patterns is a property of the second-stage filters in some FRF models, these models do not predict that the same neurons should respond selectively to both first- and second-order patterns.

The neurophysiology of human second-order vision has been studied with functional MRI (fMRI) and PET. Most studies to date have focused on second-order visual motion processing (Dumoulin et al. 2003; Dupont et al. 2003; Nishida et al. 2003; Seiffert et al. 2003; Smith et al. 1998; Wenderoth et al. 1999), with somewhat conflicting results. While all of these studies found that first- and second-order motion evoked responses in largely the same regions of visual cortex, only some of them found stronger activation by one stimulus type in any cortical area (as might be expected if the neurons within a particular cortical area were predominantly responsive to a single stimulus category). Smith et al. (1998) reported that second-order motion evoked stronger responses in areas V3 and $\mathrm{V} 3 \mathrm{~A} / \mathrm{B}$ than did first-order motion. Wenderoth et al. (1999), using PET, reached a similar conclusion. In contrast, Dumoulin et al. (2003) found no difference between the fMRI responses to first- and second-order motion in V3 and V3A/B, but instead observed significantly stronger responses to firstorder motion in V1 and stronger responses to second-order motion in lateral occipital cortex anterior to V3 (but not including V5/MT). Other studies failed to find any significant differences between first- and second-order motion (Dupont et al. 2003; Nishida et al. 2003; Seiffert et al. 2003).

With the exception of the study by Nishida et al. (2003), the studies cited above relied on finding overall response differences between two physically different stimulus categories (e.g., stimulus plus carrier vs. carrier alone, or 1st- vs. 2ndorder stimuli). A lack of a difference in the overall level of response, however, does not necessarily imply a lack of stimulus selectivity. For example, if one subpopulation of neurons responds only to first-order stimuli and a separate subpopulation of intermingled neurons responds equally strongly only to second-order stimuli, there will be no difference in the overall response (averaged across both subpopulations) to first- and second-order stimuli. The two subpopulations could, however, be distinguished using an adaptation protocol. Response adaptation provides a means for revealing separate subpopulations of neurons selectively tuned for different stimuli even when these neurons are intermingled at a spatial scale that is smaller than the sampling resolution (voxel size) of the measurements. A sampled region of tissue containing neurons selectively tuned for one stimulus category will adapt-i.e., respond less-after repeated presentation of these neurons' preferred stimulus. If the same tissue contains a second, separate subpopulation of neurons selectively tuned for a different stimulus category, that tissue will also adapt to that stimulus category. Repeated presentation of one stimulus category will not, however, affect the postadaptation responses to the other stimulus category. Selective adaptation to a particular stimulus category 
thus provides a measure of the stimulus selectivity of a subpopulation of neurons that is unaffected by the stimulus selectivity of other neurons in the same region of tissue. The only neuroimaging study of second-order motion perception that used adaptation is that by Nishida et al. (2003), who measured direction-selective fMRI response adaptation to identify neurons tuned to first- and second-order motion direction. Although the authors found evidence of adaptation as early as V1, they failed to find any difference between the amount of adaptation to first- and second-order motion, leading them to conclude that the two types of motion are analyzed in the same cortical regions. They did not, however, test whether adapting to first-order motion influenced the responses to second-order motion or vice versa.

The pathways and visual areas processing motion are not identical to those processing static patterns and complex shapes. The study by Nishida et al. (2003), for instance, was designed to localize neurons selective for the direction of second-order motion; neurons selective for static properties of second-order patterns (e.g., orientation), but not selective for motion direction, would not have been identified by the experimental procedure used. For these reasons, studies of secondorder motion are of limited use for understanding the mechanisms underlying static second-order pattern perception. Only a small number of human neuroimaging studies have investigated perception of static second-order textures. Using fMRI, Kastner et al. (2000) found that stimuli containing texture boundaries evoked stronger responses in higher-tier extrastriate visual areas than did stimuli with uniform texture. GrillSpector et al. (1998a) studied cue invariance in object-selective cortical regions using stimuli defined, among other cues, by second-order texture boundaries. Cue-invariant responses were observed in the lateral occipital complex (LOC), but except for a small region anterior to V3, not in early retinotopic areas.

Although these earlier studies of static texture perception identified brain areas that responded to second-order patterns, they did not reveal whether any of these areas are selective for second-order stimuli. For instance, as mentioned above, psychophysical studies have shown that second-order (and 1storder) mechanisms are orientation-selective, implying that the underlying neuronal mechanisms are also orientation-selective. As a consequence, it should be possible to localize the neuronal populations mediating second-order pattern perception by identifying brain regions that exhibit orientation selectivity for second-order stimuli.

In this study, we used orientation-selective adaptation as a tool to localize populations of neurons selective for the orientation of first-order patterns (defined by luminance modulations), neurons selective for the orientation of second-order patterns (defined by carrier contrast or orientation), or cueinvariant neurons selective for the orientation of both first- and second-order patterns. We used an experimental design similar to that previously used to measure adaptation in electrophysiology and psychophysics experiments (Bradley et al. 1988; Carandini et al. 1997, 1998; De Valois 1977; Hammett and Snowden 1995; Kohn and Movshon 2003; Pantle and Sekuler 1968; Sclar et al. 1989; Snowden and Hammett 1996; Solomon et al. 2004). Importantly, we used a highly demanding foveal task to divert attention away from the stimuli to equate spatial attention across stimulus conditions. We found orientationselective response adaptation to both first- and second-order patterns in multiple visual areas, including V1, with no single visual area specialized for either stimulus type. Most of the response adaptation we observed for first-order stimuli could be accounted for by adaptation in V1 neurons. Response adaptation to second-order stimuli, on the other hand, was significantly stronger in several extrastriate visual areas than in V1, particularly ventral area VO1, implying that the proportion of neurons selective for second-order pattern orientation was greater in these areas than in V1. We did not find convincing evidence for cue-invariant orientation-selective response adaptation; adaptation to first-order stimuli did not consistently reduce the responses to second-order stimuli in any visual area examined. Our results are consistent with an FRF model in which the second filtering stage is mediated by neurons primarily in ventral extrastriate visual areas.

\section{METHODS}

Three subjects (the authors), between 37 and $48 \mathrm{yr}$, participated in the experiment. Subjects gave informed consent to participate in accordance with the Helsinki convention and National Institutes of Health guidelines for human subject experiments. The experiments were approved by the local Human Subjects Committee of New York University. The experiments were undertaken in compliance with the safety guidelines for MRI (Kanal et al. 2002).

\section{Stimulus conditions}

We measured the postadaptation fMRI responses to presentations of vertical or horizontal grating patterns (see Visual stimuli for a detailed description) with modulations of either luminance (condition LM:LM; 1st-order), carrier contrast (condition CM:CM; 2nd-order), or carrier orientation (conditions OM:OM and LM:OM; 2nd-order). In the unimodal adaptation conditions LM:LM, CM:CM, and OM:OM, the adapter and probe patterns were of the same stimulus type. In the cross-modal adaptation condition LM:OM, the adapter pattern was first-order but the probe pattern second-order. The conditions differed only in the types of stimuli used, but were otherwise identical in design. Each subject underwent two scanning sessions per condition, one for each adapter orientation (horizontal or vertical). The results for different adapter orientations were pooled to compensate for any orientation bias in the responses.

\section{Adaptation protocol}

We used an event-related design modeled on psychophysical and electrophysiological adaptation experimental protocols (Bradley et al. 1988; Carandini et al. 1997, 1998; De Valois 1977; Hammett and Snowden 1995; Kohn and Movshon 2003; Pantle and Sekuler 1968; Sclar et al. 1989; Snowden and Hammett 1996; Solomon et al. 2004) to measure the average response to single presentations of an intermediate-contrast probe stimulus after adaptation to a high-contrast adapter stimulus (the actual contrasts used are listed below). The trial structure is shown in Fig. $2 A$. Before scanning, at the beginning of each experiment, subjects passively viewed the adapter stimulus for $100 \mathrm{~s}$. Each subsequent trial had a duration of $7.2 \mathrm{~s}$. Adaptation was maintained by showing a "top-up" adapter during the first $4 \mathrm{~s}$ of each trial. The top-up adapter was followed by a blank screen for $1 \mathrm{~s}$, which was in turn followed by presentation of the probe stimulus for $1 \mathrm{~s}$. The trial ended with a 1.2-s display of a blank screen (uniform gray except for the fixation point). Throughout the trial, subjects performed a highly attention-demanding task at fixation, thus ignoring the adapter and probe stimuli. A single adapter orientation was used for each scanning session. (A note on terminology: we use the term "scan" to refer to a single fMRI data collection run, typically about 5 min long, 

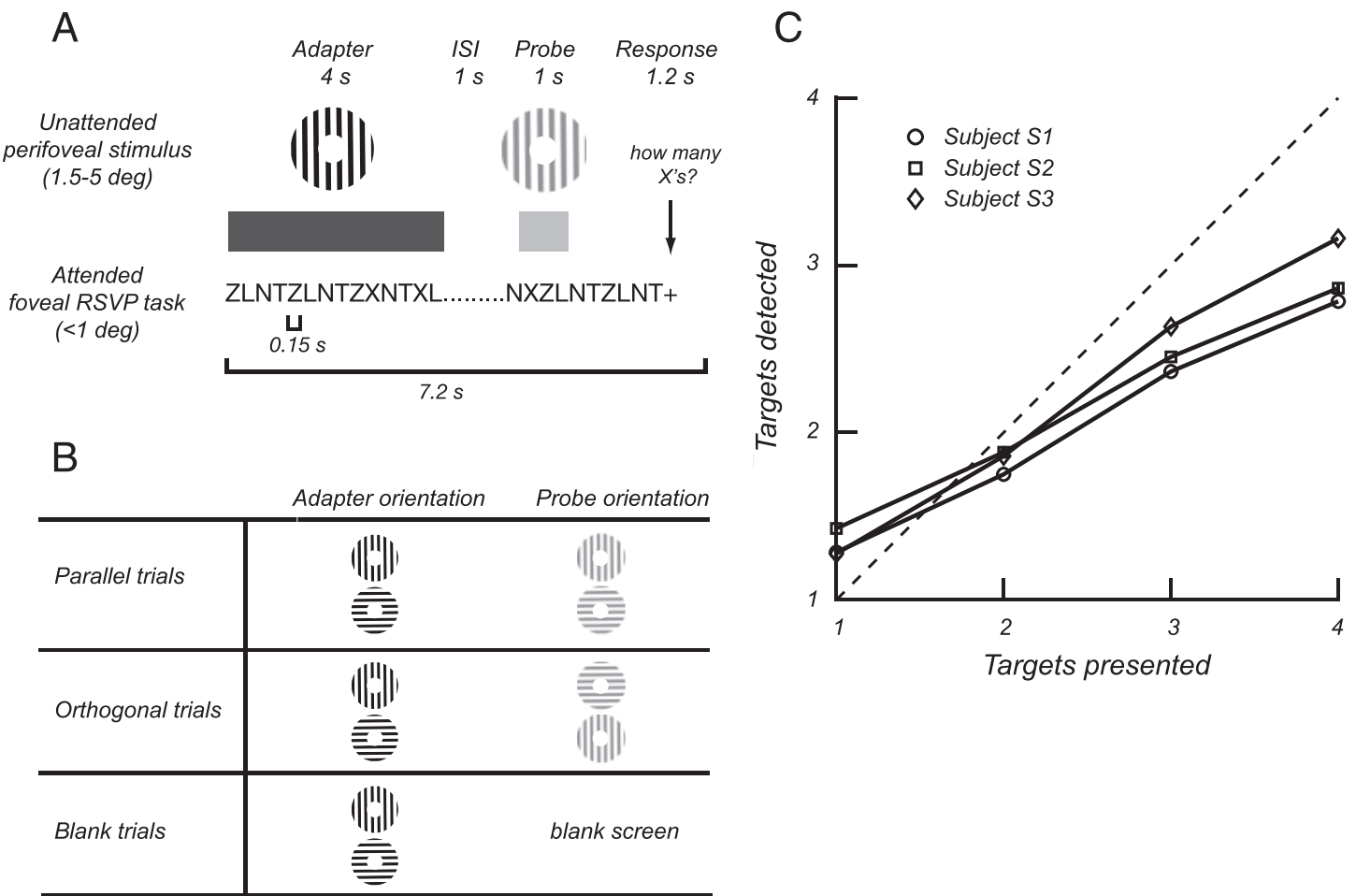

FIG. 2. A: structure of a single functional MRI (fMRI) trial. Adapter and probe stimuli (1st- and 2nd-order gratings) were displayed within an annulus around the center of fixation while subjects performed a highly attention-demanding rapid serial visual presentation task at fixation (counting the number of target letters $\mathrm{X}$ in a rapid stream of distractor letters Z, L, N, and T). Each trial consisted of a top-up, high-contrast adapter stimulus for $4 \mathrm{~s}$ (indicated by dark gray bar), followed by a blank screen for $1 \mathrm{~s}$, followed by an intermediate-contrast probe stimulus for $1 \mathrm{~s}$ (indicated by light gray bar). Trials ended with a blank screen for $1.2 \mathrm{~s}$, during which time subjects responded how many targets they had seen by a button press. $B$ : trial types. On parallel trials, orientation (horizontal or vertical) of probe stimulus was the same as orientation of adapter stimulus. On orthogonal trials, probe stimulus was orthogonal to adapter stimulus. On blank trials, only adapter stimulus was shown, and probe stimulus was replaced by a blank screen for the same duration (1 s). C: performance of subjects on RSVP task. Diagonal line indicates perfect performance; chance level performance would correspond to a horizontal line. Error bars (SE) are smaller than plot symbols.

and "session" to refer to a set of scans run in direct succession.) The spatial phase of the adapter and probe stimuli was varied randomly at $4 \mathrm{~Hz}$. In one-third of the trials, the probe stimulus had the same orientation as the adapter (parallel or adapted trials); in one-third, the probe stimulus was perpendicular to the adapter (orthogonal or unadapted trials); and in one-third of the trials, the screen remained blank throughout the probe stimulus presentation phase (blank trials; Fig. 2B). A scanning session was comprised of 2 localizer scans and 10 adaptation scans, each consisting of 42 trials (14 trials of each of the 3 trial types). The trials were pseudorandomized such that the sequence of trials preceding and following any trial was equally likely to contain any of the three trial types. Specifically, the 14 trials of each trial type were presented in seven blocks of 6 trials each, with trial order randomized within blocks. The large number of trials (420 trials per session) made any systematic biases caused by trial order very unlikely.

\section{Attentional control task}

It is well known that spatial attention can strongly modulate the neuronal responses to visual stimuli measured with fMRI in a spatially specific manner, confounding the interpretation of the results (Huk et al. 2001). To control and equate attentional load across conditions, we used a highly attention-demanding task at the center of fixation that was identical across trials and conditions (Fig. 2A). The attentional control task required subjects to count the number of target letters (Xs) shown in a stream of rapidly presented distractor letters (10 repeated cycles of Z-L-N-T, in that order, each presented for $150 \mathrm{~ms}$ ) and report the number of targets observed at the end of each trial. The letters were shown continuously for $6 \mathrm{~s}$ throughout each trial from the beginning of presentation of the adapter until the end of presentation of the probe stimuli. After $6 \mathrm{~s}$, the letters were replaced by a fixation cross, cueing subjects to respond by pressing one of four keys corresponding to the number of target Xs presented (1-4). The targets could appear at any time throughout the trial, but two targets could not appear in direct succession. Although in theory this implied that, on a small proportion of trials, all targets could appear at the beginning of a trial, making it unnecessary for subjects to maintain attention at the center of gaze for the remainder of the trial, in practice the task was so difficult that subjects were never certain that they had seen all targets. Despite the great attentional demands of the task, subjects' performance after practice was well above chance level (Fig. 2C). Informally, we could verify that the task was highly effective at diverting attention from the periphery, because subjects reported that they were unable to perceive the orientation of the probe stimuli while performing the task, whereas with attention directed to the probe stimuli, discriminating the orientation of the probe stimuli was trivial.

\section{Psychophysical measurement of adaptation}

To verify that the adapter stimuli were effective in eliciting adaptation, we measured psychophysical postadaptation detection thresholds to stimuli under conditions similar to those in the fMRI experiment. For this experiment, the attentional control task was replaced by a two-interval forced choice task that involved determining which of two sequentially presented probe patterns contained a (1st- or 2ndorder) target stimulus. Before each session, subjects viewed the adapter stimulus for $100 \mathrm{~s}$, analogous to the fMRI experiments. Each trial was $6.6 \mathrm{~s}$ long and began by presenting a top-up adapter for $4 \mathrm{~s}$, followed by a blank screen for $0.5 \mathrm{~s}$, followed by two stimuli for $0.5 \mathrm{~s}$ each, separated by an interstimulus interval (ISI) of $0.5 \mathrm{~s}$. On one-half of the trials, the first interval contained the target, whereas on the other half of trials, the target was presented in the second interval. Nontar- 
get stimuli were generated in the same way as the target stimuli, but with modulation amplitude set to zero. At the end of the trial, subjects had $1.1 \mathrm{~s}$ to respond which interval contained the target. The modulation depth of the target stimulus was varied by a one-up, two-down staircase procedure, with two interleaved staircases. A single experimental session consisted of 10 blocks, each consisting of 20 trials. Target orientation alternated from block to block but was constant within a block to minimize perceptual interactions between the carrier stimulus and the modulator (Morgan et al. 2000). A single adapter orientation was used for each session, and sessions were run on different days to avoid potential confounding effects of long-term adaptation. One hundred trials were run for each stimulus condition (LM:LM, CM:CM, OM:OM, and LM:OM), trial type (adapt orthogonal, adapt parallel), and adapter orientation (vertical and horizontal). The results were pooled across adapter orientations, and psychometric functions were fitted to the data using a bootstrap procedure (Wichmann and Hill 2001a,b). Detection thresholds were defined as the modulation contrast corresponding to $75 \%$ correct detection.

\section{Visual stimuli}

The stimuli were sinusoidally modulated horizontal or vertical gratings presented within an annulus with inner radius $1.5^{\circ}$ and outer radius $5^{\circ}$ around the center of fixation (Fig. 3). The modulation spatial frequency was $1.5 \mathrm{cpd}$ for all stimulus types.

In condition LM:LM, gratings (Fig. $3 A$ ) were generated by modulation of the background luminance

$$
\begin{gathered}
L_{\mathrm{LM}}(x, y)=L_{0}\left[1+A_{\mathrm{M}} M(x, y)\right], \\
M(x, y)=\left\{\begin{array}{lc}
\sin (2 \pi f x+\phi) & \text { (vertical modulator) } \\
\sin (2 \pi f y+\phi) & \text { (horizontal modulator) }
\end{array}\right.
\end{gathered}
$$

where $L_{\mathrm{LM}}$ is the grating stimulus, $L_{0}$ the background luminance, the modulator $M$ is a two-dimensional horizontal or vertical sine wave grating with spatial frequency $f$ and phase $\phi$, and $A_{\mathrm{M}}$ is the modulation amplitude (peak contrast). The modulation amplitudes of the adapter and probe stimuli were 80 and $10 \%$, corresponding to root-meansquare (RMS) contrasts of 57 and $7 \%$, respectively.

In condition $\mathrm{CM}: \mathrm{CM}$, grating patterns (Fig. $3 B$ ) were generated by modulating the luminance contrast of a noise carrier pattern $N(x, y)$

$$
L_{\mathrm{CM}}(x, y)=L_{0}\left[1+\frac{1}{2}\left(1+A_{\mathrm{M}} M(x, y)\right) N(x, y)\right] .
$$

The carrier was generated by filtering zero mean binary (with values -1 or 1) random noise with an isotropic band-pass filter having a center spatial frequency of $6 \mathrm{cpd}$ and a bandwidth of one octave. Carrier RMS contrast was $25 \%$. The modulation amplitude $A_{\mathrm{M}}$ was $100 \%$ for the adapter and $60 \%$ for the probe stimuli. A small number of pixels had values smaller than -1 or $>1$ (corresponding to the lowest and highest luminance of the display hardware); these were clipped at -1 or 1 , respectively. We used a higher modulation amplitude for the second-order stimuli to compensate for the higher detection thresholds of second-order patterns (e.g., Landy and Oruç 2002) and roughly equate the salience of different stimulus types.

In condition OM:OM, two noise carriers were used that had the same spatial frequency and contrast as in condition CM:CM, but were oriented either horizontally $\left(N_{\mathrm{h}}\right)$ or vertically $\left(N_{\mathrm{v}}\right)$ with an orientation bandwidth of $30^{\circ}$. Second-order grating patterns (Fig. $3 C$ ) were generated by mixing the two oriented carriers and modulating the relative amount of each carrier in a sinusoidal fashion (Landy and Oruç 2002)

$L_{\mathrm{OM}}=L_{0}\left[1+\left(\frac{1}{2}\left(1-A_{\mathrm{M}} M(x, y)\right)\right)^{0.5} N_{\mathrm{v}}(x, y)+\left(\frac{1}{2}\left(1+A_{\mathrm{M}} M(x, y)\right)\right)^{0.5} N_{\mathrm{h}}(x, y)\right]$.

The square roots in this equation served to keep expected contrast energy constant across the stimulus to ensure that the stimuli in this condition could only be detected by a mechanism sensitive to carrier orientation modulation. In other words, these stimuli would be invis-
A

$L M$

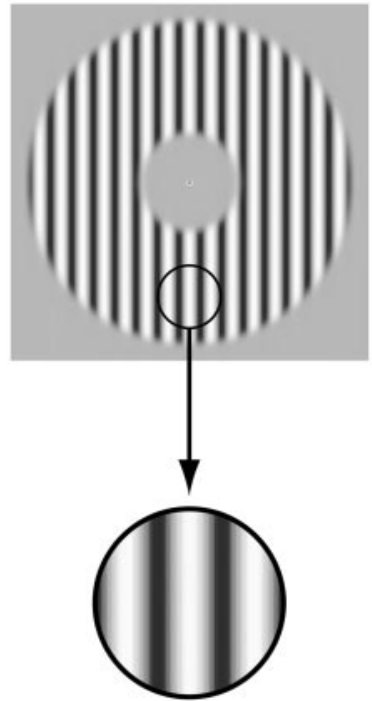

B

CM

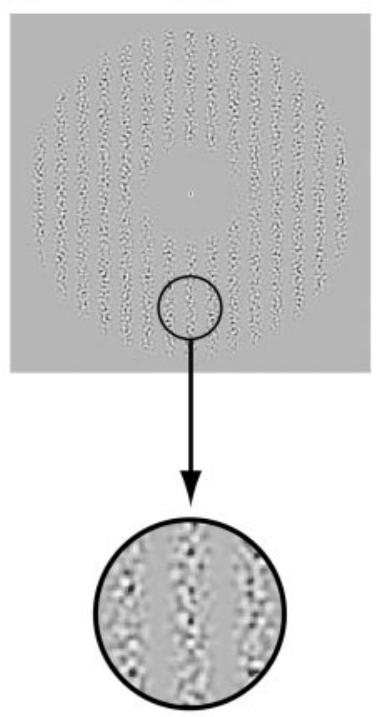

C

$O M$

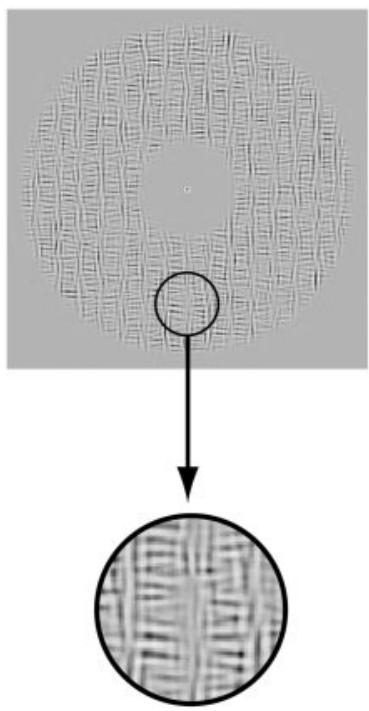

D LM:OM

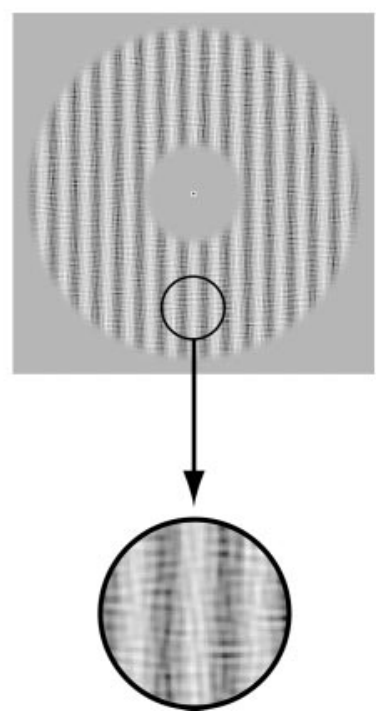

FIG. 3. Examples of vertical adapter stimuli. $A$ : luminance modulated (LM) grating (condition LM:LM). $B$ : contrast modulated (CM) grating (condition CM:CM). C: orientation modulated (OM) grating (condition OM:OM). D: luminance modulated grating superimposed on oriented noise carrier (condition LM:OM). Stimulus contrast has been adjusted to improve visibility for printing. Stimulus annulus had an outer radius of $5^{\circ}$ and an inner radius of $1.5^{\circ}$ from the center of fixation. Enlargements of portions of stimuli are shown below.

ible to a mechanism sensitive only to contrast modulations (whereas such a mechanism would respond optimally to the stimuli in condition CM:CM). The modulation amplitudes $A_{\mathrm{M}}$ of the adapter and probe stimuli were 100 and $85 \%$, respectively.

In condition LM:OM, the (2nd-order) probe stimulus was identical to that of condition OM:OM, but the (1st-order) adapter stimulus (Fig. $3 D$ ) was generated by superimposing a luminance grating on an equal mix of horizontal and vertical noise carriers (identical to those used in condition OM:OM)

$$
L_{\mathrm{LM}: \mathrm{OM}}(x, y)=L_{0}\left[1+\frac{1}{2}\left(N_{\mathrm{v}}(x, y)+N_{\mathrm{h}}(x, y)\right)+A_{\mathrm{M}} M(x, y)\right] .
$$

The adapter modulation depth $A_{\mathrm{M}}$ in this condition was $50 \%$. The rationale for adding the carrier pattern to the first-order adapter 
A

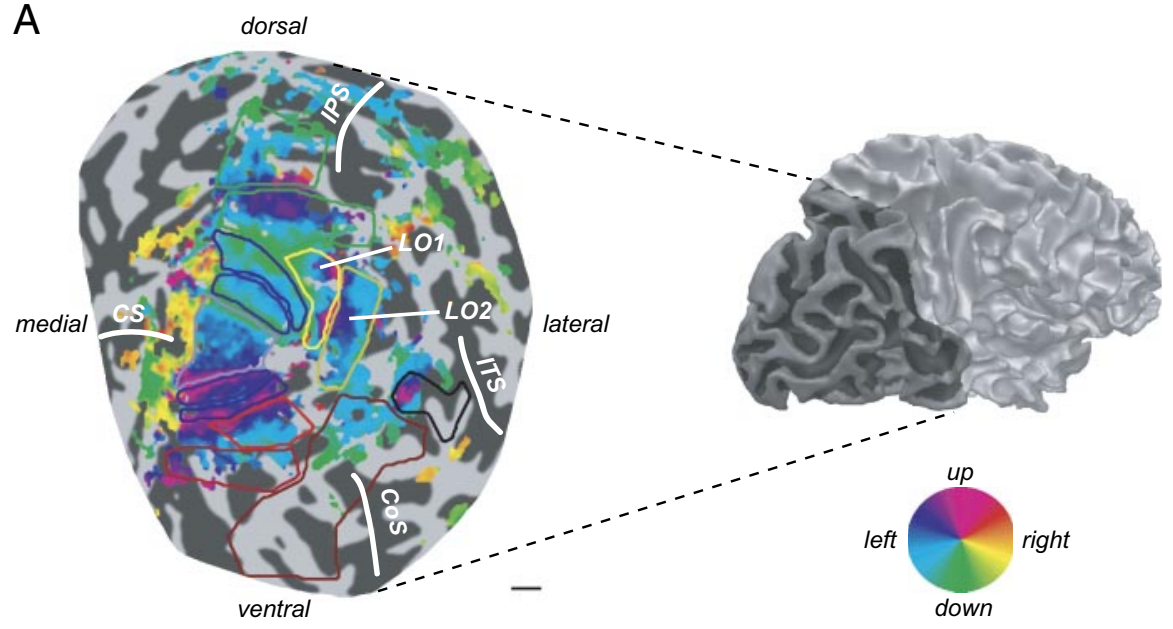

FIG. 4. A: flat map of visual polar angle representation (thresholded at a coherence $>0.25$ ) overlaid on a patch of flattened cortex from the right hemisphere of subject S2, with boundaries of visual area regions of interest (ROIs) superimposed. Except for areas V5/MT and posterior lateral occipital complex (pLOC), ROIs have been restricted to the eccentricity range $\left(1.5-5^{\circ}\right)$ corresponding to the stimulus annulus in the current experiment. Flat map corresponds to region of cortex indicated by dark gray on the 3-dimensional rendered brain to the right. Colors indicate polar angle in the visual field (color scale on bottom right). Polar angles in LO1 and LO2 cover the full color range from red to green, evidence that each of these areas contains a map of the entire contralateral hemifield. Scale bar: $1 \mathrm{~cm}$ (note that this is an average

B

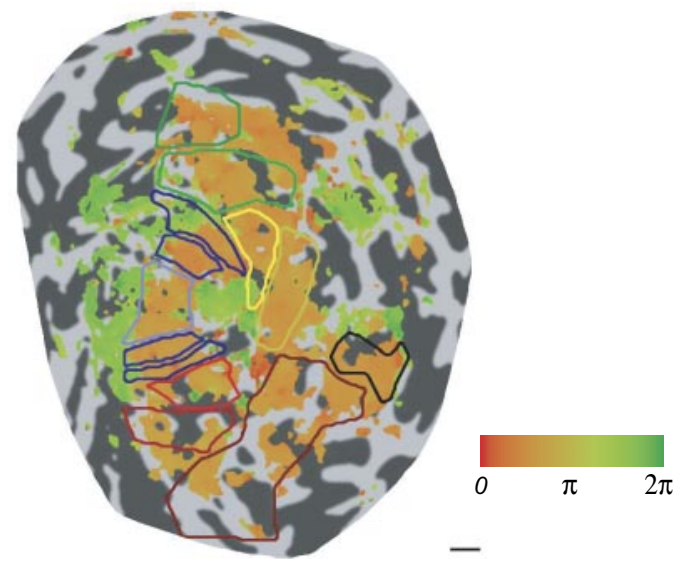
value; because of inevitable distortions in the flattened representation, scale can vary $\leq 50 \%$ from the center to the periphery.) CS, calcarine sulcus; IPS, intraparietal sulcus; ITS, inferior temporal sulcus; $\mathrm{CoS}$, collateral sulcus. $B$ : fMRI response evoked by localizer stimulus in condition CM:CM for same subject and hemisphere as in $A$. Red-green colors represent phase of response for voxels with a coherence $>0.2$. Phases between 0 and $\pi$ (indicated by shades of red) correspond to stimulus present blocks (activations); phases between $\pi$ and $2 \pi$ (indicated by shades of green) correspond to stimulus absent blocks (deactivations).

stimulus in this condition, rather than a pure luminance adapter as in condition LM:LM, was to equate the amount of carrier adaptation, and thus the baseline carrier response amplitude, between conditions OM:OM and LM:OM.

The same stimuli were used both in the psychophysical and in the fMRI experiments, except that to determine detection thresholds, the modulation depth of the probe stimuli was varied over a sevenfold range spanning the detection thresholds as verified by pilot runs. Stimuli were presented at $800 \times 600$-pixel resolution on an electromagnetically shielded analog NEC2110 LCD display (for the fMRI experiments) or a Nokia 446 XPro CRT display (for psychophysics) using the Psychophysics Toolbox (Brainard 1997; Pelli 1997) and a 10-bit graphics card. Both displays had a refresh rate of $60 \mathrm{~Hz}$. The displays were carefully calibrated to minimize potential first-order artifacts caused by nonlinearities in the display hardware.

\section{Definition of visual area regions of interest}

Nine regions of interest (ROIs) were defined based on retinotopy, and two additional ROIs (V5/MT+ and LOC) were defined by a combination of retinotopy and functional properties. Standard traveling wave methods for retinotopic mapping were used to identify boundaries between retinotopically organized visual areas (DeYoe et al. 1996; Engel et al. 1994; Sereno et al. 1995). Area boundaries were identified as the phase reversals (corresponding to representations of the horizontal and vertical visual field meridians) in a map of the polar angle representation of the visual field, measured by the phase of the fMRI response to a slowly $(0.04 \mathrm{~Hz})$ rotating wedge stimulus $\left(45^{\circ}\right.$ wide) extending from the center of gaze to $6^{\circ}$ eccentricity. Eccentricity was mapped in a similar fashion by measuring the phase of the

response to a slowly expanding or contracting stimulus annulus (width $1.5^{\circ}$, corresponding to a duty cycle of $25 \%$ ). The retinotopic mapping scans were carried out in separate scanning sessions for each subject.

We identified nine retinotopically organized areas in every subject (shown for the right hemisphere of subject S2 in Fig. 4A). We have reliably identified these nine cortical areas consistently in a total of 12 subjects, including the 3 subjects participating in this study. Most of these areas-V1, V2, V3, V3A, V3B, V7, hV4, and VO1- have been previously described in the literature (DeYoe et al. 1996; Press et al. 2001; Sereno et al. 1995; Smith et al. 1998; Tootell et al. 1995, 1997, 1998a; Wade et al. 2002; Wandell et al. 2005). VO1 is a coarsely retinotopic area anterior and lateral to hV4 (Wandell et al. 2005), which may correspond to area TEO as described by Kastner et al. (2000). In addition, we have identified two retinotopic areas in the lateral occipital cortex between dorsal V3 and V5/MT+ not previously described in the literature, although one of these areas may overlap V3B as originally described (Smith et al. 1998). The detailed retinotopic organization and functional characteristics of these areas, which we have termed LO1 and LO2 (for lateral occipital areas 1 and 2), will be reported elsewhere. For the purposes of this study, we will only provide a brief description of their location and retinotopic organization, focusing instead on their response properties in the context of second-order pattern perception. We have provided the retinotopic maps of the three subjects as supplementary on-line material. ${ }^{1}$

V3B was initially described as an incomplete map of the contralateral hemifield located anterior to dorsal V3 (Smith et al. 1998). Later

\footnotetext{
${ }^{1}$ The Supplementary Material for this article (3 maps) is available online at http://physiology.org/cgi/content/full/00668.2005/DC1.
} 
papers described it as containing a full hemifield map, but adjacent to and sharing a fovea with V3A (Press et al. 2001; Wandell et al. 2005). Although we found evidence of a separate visual field map sharing a fovea with V3A that matches the description of V3B by Press et al. (2001) and Wandell et al. (2005), the boundaries of this map were not sufficiently consistent to separate it from V3A in every subject. Hence, to indicate that our V3A ROI also includes V3B as defined by Press et al. (2001), we have labeled this ROI V3A/B.

The area that we have named LO1 is a complete map of the contralateral visual hemifield extending from the anterior boundary of dorsal V3 and sharing the central foveal confluence of areas V1, V2, and V3 (Fig. 4A). Visual field eccentricity in this map extended in the same direction as in dorsal V3, i.e., with peripheral locations represented anteriorly and dorsally. This map differed from V3B described by Smith et al. (1998) in two significant ways. First, the original description of V3B did not explicitly comment on the representation of eccentricity within the map, making exact comparison with later studies difficult. Second, Smith et al. (1998) found only a representation of the lower quadrant in this area, whereas we found a representation of the entire contralateral hemifield. Because this map did not match any previously described visual area, we have named this area LO1, for lateral occipital area 1 . We have used the nomenclature proposed by Wandell et al. (2005), by which areas are named by gross anatomical location and a unique number.

In addition to LO1, we reliably identified in every subject a previously undescribed retinotopic area anterior to LO1 which we have named area LO2 (Fig. 4A). Like LO1, LO2 contained a full map of the contralateral visual hemifield and shared the central foveal representation of $\mathrm{V} 1, \mathrm{~V} 2$, and $\mathrm{V} 3$. The polar angle representation in LO2 was the mirror image of that in LO1, with the upper vertical meridian (defining the boundary with LO1) represented caudally and the lower vertical meridian represented rostrally. Visual field eccentricity in LO2 was mapped parallel to that of LO1, with the visual field periphery mapped anteriorly and dorsally. LO2 was posterior to and did not overlap with functionally defined area V5/MT + . Based on retinotopic criteria, we suggest LO2 should be considered a separate visual area. Furthermore, responses in LO2 to images of intact objects and faces were larger than responses to scrambled images and faces, suggesting that LO2 formed part of the object-selective LOC. Our results also show that the response properties of LO2 with regard to first- and second-order patterns differed markedly from those of LO1 and more posterior visual areas.

We also ran a separate session for each subject to delineate ROIs comprising functionally defined areas V5/MT + , based on its stronger response to random moving dots than to stationary dots (Huk et al. 2002; Tootell et al. 1995; Watson et al. 1993), as well as the LOC, based on its stronger response to images of intact objects and faces than to images of scrambled objects and faces (Grill-Spector et al. 1998b; Kourtzi and Kanwisher 2000, 2001; Lerner et al. 2002; Malach et al. 1995). These scans were run using a block design, alternating ten 12-s-long stimulus blocks (moving dots for V5/MT+, images of intact objects for LOC) with ten 12-s baseline blocks (stationary dots for $\mathrm{V} 5 / \mathrm{MT}+$, scrambled images of objects for LOC). Because both of these contrasts also activated parts of retinotopic areas, we restricted the V5/MT+ and LOC ROIs to exclude retinotopic visual areas. Most of the anterior part of the LOC ROI did not overlap with regions activated by the stimuli in the adaptation scans (as assessed with independent localizer scans), and so we further restricted this ROI to include only the posterior section (excluding retinotopic areas). We refer to this ROI as posterior LOC or pLOC in the following. Consistent with previous work (Huk et al. 2002), we found that functionally defined V5/MT+ could be subdivided into a posterior retinotopically organized part (putative human V5/MT) and an anterior nonretinotopic part (putative human MST), but for the purposes of this study, we did not consider these subdivisions separately.

\section{Localizer scans}

In each scanning session, before and after the series of adaptation scans, we measured responses to the probe stimuli presented alone (i.e., without the adapter stimuli), to independently identify the cortical regions responding to the stimulus patterns. The stimuli in the localizer scans were the same as the probe stimuli used in the adaptation scans for a given session. Thus the localizer scan stimuli in the LM:LM condition were the LM probe gratings, whereas in the LM:OM condition, the stimuli were the OM probe gratings. For these scans, we used a block design, alternating 9.6-s on blocks of the intermediate-contrast probe stimuli, randomly changing in orientation and phase at $4 \mathrm{~Hz}$, with 9.6-s OFF blocks of blank screen (uniform gray except for the fixation point). Subjects were instructed to maintain their gaze on a fixation marker throughout the scan. Each localizer scan consisted of 10 stimulus-blank alternations.

\section{MRI acquisition}

Experiments were carried out on a Siemens Allegra 3T scanner, equipped with a four-channel phased-array surface coil covering the back of the head (NM-011 transmit head coil and NMSC-021 receive coil, Nova Medical, Wakefield, MA). A custom-fitted bitebar was used to minimize subject head motion. Standard echoplanar imaging methods were used to measure the blood oxygenation level-dependent (BOLD) signal (Ogawa et al. 1990) in T2*-weighted images. Functional data in the adaptation scanning sessions were acquired using the following parameters: TR 1,200 ms; TE $30 \mathrm{~ms}$; flip angle $75^{\circ} ; 64 \times 64$ matrix size; 19 slices oriented perpendicular to the calcarine sulcus; voxel size $3 \times 3 \times 3 \mathrm{~mm}$. For the retinotopy, $\mathrm{V} 5 / \mathrm{MT}+$, and LOC sessions, we used the same imaging parameters with the following exceptions: 24 slices and 1,500-ms TR. At the beginning of each session, we also acquired an anatomical T1weighted MPRAGE image that covered the same volume as the functional scans, but with twice the in-plane resolution (voxel size $1.5 \times 1.5 \times 3 \mathrm{~mm}$ ). This image was used to compute the alignment between the functional volumes and the high-resolution anatomical image used to extract cortical surfaces, using an automated robust image registration method (Nestares and Heeger 2000). The alignment parameters obtained were used to project the visual area ROIs (defined in the high-resolution image space) into the image space of each functional scan. To visualize the fMRI responses from the localizer and retinotopy measurements, the statistical data were projected onto the flattened occipital cortex, but no quantitative analyses were performed on the flattened data. By analyzing our data in the native functional image space rather than aligning the data itself to a standard space, we minimized blurring that would have been introduced through interpolation.

\section{fMRI data analysis}

The time series data for each scan were corrected for motion within and between scans using MCFLIRT (Jenkinson et al. 2002). The estimated head movements were consistently $<1 \mathrm{~mm}$ in any direction. We also manually inspected each time series to ensure there were no sudden movements or artifacts in the data.

Data from the localizer scans, the V5/MT+ scans, and the LOC scans were analyzed separately for every voxel by correlating the time series data with a sinusoid at the stimulus alternation frequency. The time series were first normalized by dividing by the mean (to compensate for variations in intensity with distance from the receiver coil) and detrended with a high-pass filter to remove low-frequency noise and drift (Biswal et al. 1995, 1997a,b; Purdon and Weisskoff 1998; Smith et al. 1999; Zarahn et al. 1997). For each voxel, we computed the correlation (technically coherence) between the best-fit sinusoid and the measured time series. This analysis also yielded a response phase and amplitude, allowing us to distinguish stimulus-correlated 
significant increases (activations) in the BOLD response from significant decreases (deactivations). Details of this analysis method have been published elsewhere (Backus et al. 2001; Huk and Heeger 2002; Neri et al. 2004; Zenger-Landolt and Heeger 2003).

Retinotopy data were analyzed using the same procedures. Because the stimuli moved progressively through the visual field, the measured response phases corresponded to (angular or radial) locations in the visual field (Fig. 4A) (DeYoe et al. 1996; Engel et al. 1994; Sereno et al. 1995). Retinotopic visual area ROIs were drawn on computationally flattened representations (flat maps) of the occipital cortex generated from high-resolution T1-weighted anatomy images using the public domain software SurfRelax (Larsson 2001).

Event-related data from the adaptation scans were analyzed for each visual area ROI as follows. First, separately for each adaptation session, the ROI was restricted to include only those voxels showing significant activation in the localizer scans carried out in the same session. Specifically, the ROIs were restricted to include voxels with a response coherence $>0.2$ and a response phase between 0 and $\pi$, corresponding to stimulus on blocks (the phase range bracketed the variation of hemodynamic delays across voxels). This ensured that our ROIs only included voxels corresponding to visual field locations within the stimulus annulus and excluded voxels that did not show an increased response to visual stimuli (e.g., deactivations). The coherence threshold was chosen to obtain a consistent size of individual ROIs across conditions and repetitions, because the strength of the evoked fMRI response varied between scanning sessions (Aguirre et al. 1998). Although we used different visual stimuli for the localizer scans for different stimulus conditions, the different stimuli covered the exact same parts of the visual field (the stimulus annulus) and the spatial extent of evoked fMRI responses were very similar for different stimulus types. Furthermore, the exact coherence threshold used was not critical. We also analyzed our data with more conservative coherence thresholds (0.3 and 0.4), and the results were consistent with those obtained with a coherence threshold of 0.2 .

For each voxel within the ROI (combined across left and right hemispheres), the raw unfiltered time-course of the fMRI response was normalized to percent signal change by dividing by the mean intensity across the scan. The normalized time-courses were averaged across voxels to yield a mean ROI time-course. Responses to individual trials were extracted from the mean ROI time-course by extracting the 16 time-points (19.2 s) starting with the onset of each trial. The mean response to the adapter stimulus alone, computed by averaging the responses to the blank trials, was subtracted from each adaptation trial (orthogonal and parallel), and the resulting time-courses were adjusted to a zero baseline by subtracting the mean of the first four time-points (before the onset of the probe stimulus). From the time-courses obtained by this procedure, we extracted a response vector $R_{\mathrm{i}}$ for each adaptation trial $i$ by extracting the eight time-points $(9.6 \mathrm{~s})$ from the onset of the probe stimulus. We computed a mean response vector $\bar{R}$ by averaging the responses for all $N$ adaptation trials regardless of trial type (orthogonal and parallel)

$$
\bar{R}=\frac{1}{N} \sum_{i=1}^{N} R_{i} .
$$

For each trial $i$ we computed a scalar response amplitude $A_{\mathrm{i}}$ by projecting the individual trial response vector $R_{\mathrm{i}}$ onto the mean response vector $\bar{R}$

$$
A_{i}=\frac{R_{i} \cdot \bar{R}}{\|\bar{R}\|} .
$$

The response amplitudes of individual trials for each trial type were averaged to compute the mean response amplitudes for each visual area ROI. The resulting response amplitudes had units of percent change in image intensity. Confidence intervals for amplitude means were calculated for individual subjects as the SE across trials. For individual subjects, we estimated the statistical significance of adaptation (meaning larger response amplitudes to orthogonal than to parallel trials) using a one-tailed $t$-test. For group means, we used a bootstrap procedure (Efron and Tibshirani 1993) to estimate significant effects of adaptation for the mean of the three subjects (not the population mean, which our sample size is too small to estimate). We estimated the underlying distribution of the mean difference between response amplitudes to orthogonal and parallel trials, averaged across subjects, by calculating the amplitude difference averaged across the three subjects for 10,000 repeated random samples taken with replacement from the original data of each subject. Each data sample was the same size as the original data set. For each resulting distribution of the mean difference, we estimated $P$ values (the probability of obtaining a null result, defined as no larger responses to orthogonal trials than to parallel trials) by the proportion of the distribution smaller than or equal to zero.

We also computed an adaptation index $I_{\mathrm{A}}$, quantifying how much the measured response changed after adaptation, relative to the overall response to the stimuli in each visual area. The index was calculated as

$$
I_{A}=\frac{A_{\perp}-A_{\|}}{\left|A_{\perp}\right|+\left|A_{\|}\right|},
$$

where $A_{\|}$is the mean amplitude of the responses to the parallel stimulus and $A_{\perp}$ the mean amplitude of the responses to the orthogonal stimulus. This index could range from -1 to 1 and was positive whenever the mean response to the orthogonal stimulus was greater than the mean response to the parallel stimulus. If the measured fMRI response to a stimulus was evoked by neurons selectively tuned to the orientation of the stimulus, and all of these neurons adapted completely (i.e., stopped responding) after presentation of the adapter stimulus, the response to the parallel probe trials would have been 0 and the adaptation index would have been 1. Intermediate degrees of adaptation and/or a smaller proportion of stimulus-selective neurons would have resulted in an adaptation index between 0 and 1 . If the neurons underlying the measured response did not adapt selectively to the stimulus orientation (even though they may have responded to the stimulus), the adaptation index would have been 0 , whereas an index smaller than 0 would have resulted if the neurons showed selective response enhancement to the adapted stimulus orientation. The use of absolute response amplitudes in the denominator ensured that the index was valid even when one or both response amplitudes was negative (corresponding to a reduction from the baseline activity level). Confidence intervals for adaptation indices were estimated using a bootstrap method (Efron and Tibshirani 1993). For each adaptation index, we estimated the underlying distribution by selecting 10,000 random samples with replacement from the original data used to compute the index. An adaptation index was calculated from each sample (each sample was the same size as the original data). Upper and lower error bounds for the mean were estimated as the 16th and 84th percentiles of the resulting distribution. Significant differences in the group mean adaptation indices between extrastriate areas and V1 (see RESULTS) were estimated for each extrastriate area from these distributions as follows. For each of the 10,000 bootstrap iterations, we calculated the mean estimated difference in the adaptation indices between V1 and the extrastriate area from the bootstrap generated distributions of adaptation indices for each subject on that iteration. This yielded a distribution of the mean difference of adaptation indices, from which we could estimate the probability of the mean difference being larger than zero.

For the group means shown in the figures below, error estimates for the mean of the three subjects were calculated as the square root of the summed squared SE for individual subjects, divided by the number of subjects. 


\section{RES ULTS}

\section{Cortical activity evoked by localizer stimuli}

For all stimulus conditions, the localizer stimuli elicited a continuous band of activity across retinotopic visual areas at the eccentricity of the stimulus. Figure $4 B$ shows the responses with a coherence threshold of 0.2 (the threshold used to restrict the ROIs for analyzing the event-related data) from the localizer scan (CM probe stimuli vs. blank) in subject S3 in condition CM:CM, overlaid on a flat map of the right hemisphere. The boundaries of the visual area ROIs are superimposed. Regions where activity increased in response to the localizer stimulus (activations) are shown in shades of orange; regions whose activity decreased in response to the stimulus (deactivations) are shown in shades of green. The spatial distribution of evoked responses across visual areas was very similar for different stimulus conditions and subjects. Particularly for early visual areas, we found a band of decreased activity peripheral and foveal to the region of increased activity. Such deactivations are commonly observed in fMRI and may partially be caused by purely hemodynamic effects (blood stealing), although there is evidence that they reflect true decreases in neuronal activity (Shmuel et al. 2002). As noted previously, deactivated voxels were excluded in the analysis of event-related data by restricting the ROIs to contain only voxels showing increases in activity to the stimulus in the localizer scans.

In lateral and ventral occipital cortex, the activity elicited by the localizer stimulus was largely restricted to the cortical regions within the visual area ROIs defined above. Our ROI analysis thus covered most or all ventral stream cortical regions that responded significantly to the stimuli. Dorsally, the activity extended beyond V7 into posterior parietal cortex along the intraparietal sulci, where additional topographically organized areas have been reported (Schluppeck et al. 2005; Silver et al. 2005). Because the slice prescription we used only partially covered these regions, dorsal regions anterior to V7 were not included in our analysis.

\section{Orientation-selective elevation of detection thresholds after adaptation}

Psychophysical detection thresholds were higher for the parallel than for the orthogonal probe stimuli, indicating orientation-selective adaptation (Fig. 5). Detection thresholds increased after adaptation for all stimulus conditions, but the threshold elevation in the cross-modal adaptation condition (LM:OM) was significant only in one of the three subjects. For subject $\mathrm{S} 2$, the threshold elevation in condition OM:OM failed to reach statistical significance by a small margin $(P<0.06)$. The shift in the psychometric functions were equivalent to a modulation contrast decrease after adaptation of $\sim 1 \%$ for LM:LM, 5-15\% for CM:CM, and 15\% for OM:OM.

\section{Event-related fMRI responses}

Robust responses to the probe stimuli in both orthogonal and parallel trials were found in most visual areas in all conditions, although responses in $\mathrm{V} 5 / \mathrm{MT}+, \mathrm{V} 7$, and pLOC were weak and quite variable. Responses to the probe in early visual areas were generally larger than those in downstream areas; mean response amplitudes in V1 were on the order of $0.8 \%$, whereas the response amplitudes in ventral higher-tier areas $(\mathrm{hV} 4$ and VO1) were $\sim 0.5 \%$ or less, and smaller still $(\sim 0.2 \%)$ in dorsal and lateral extrastriate visual areas (V3A/B, LO1, LO2). Individual subject time-courses for the V1 ROI in the LM condition are plotted in Fig. 6, together with the mean time course of the three subjects. Although the response amplitudes and the shape of the elicited response differed substantially across subjects, the response to the orthogonal (nonadapted) stimulus was stronger than the response to the parallel (adapted) stimulus in all subjects. For the V1 ROI in the LM:LM condition, the response difference to parallel versus orthogonal probe stimuli was statistically significant in two of the three subjects. Unless stated otherwise, the significant effects reported below were statistically significant both when averaged across all subjects and for at least two of the three individual subjects.

\section{Orientation-selective fMRI response adaptation}

ADAPTATION TO FIRST-ORDER STIMULI (CONDITION LM:LM). Most visual areas exhibited orientation-selective response adaptation to first-order (luminance) gratings: the responses to the orthogonal (unadapted) stimulus in the LM:LM condition were significantly stronger than the responses to the parallel (adapted) stimulus (Table 1). There was no significant response adaptation in areas $\mathrm{V} 5 / \mathrm{MT}+, \mathrm{V} 7, \mathrm{LO} 2$, and pLOC, and these areas also showed the weakest responses to the stimuli. Figure 7 shows the group-averaged time-courses in condition LM:LM
A

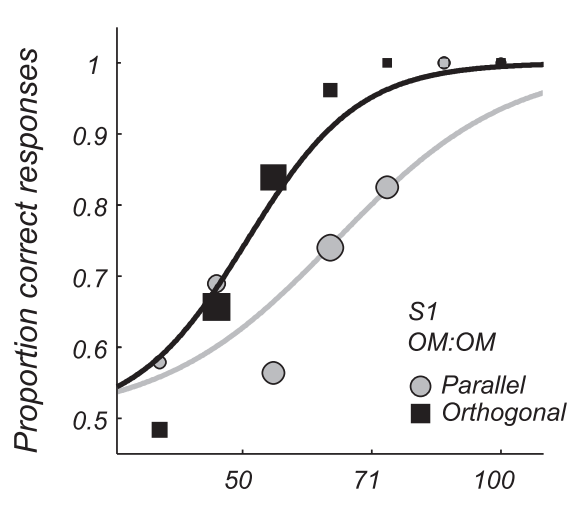

Probe contrast (\% modulation)
B

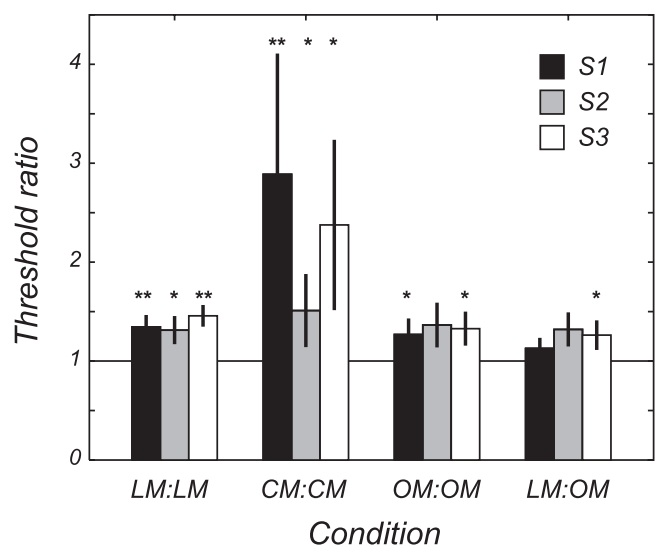

FIG. 5. Psychophysical measurements of orientation-selective adaptation. A: examples of psychometric functions fitted to detection threshold data for orientation modulated $(\mathrm{OM})$ 2nd-order gratings after adaptation to parallel (light gray curves) or orthogonal (black curves) high-contrast gratings for subject S1. Size of plot symbols is proportional to the number of data points at each probe modulation contrast. $B$ : ratios between postadaptation detection thresholds (defined by modulation contrast corresponding to $75 \%$ correct responses) measured with probe stimuli parallel to adapter stimuli and detection thresholds measured with probe stimuli orthogonal to adapter stimuli, for subjects S1-S3. Stimulus conditions are abbreviated as in Fig. 3. Asterisks indicate statistically significant threshold elevations for parallel probe stimuli $(* P<0.05 ; * * P<0.01)$. 


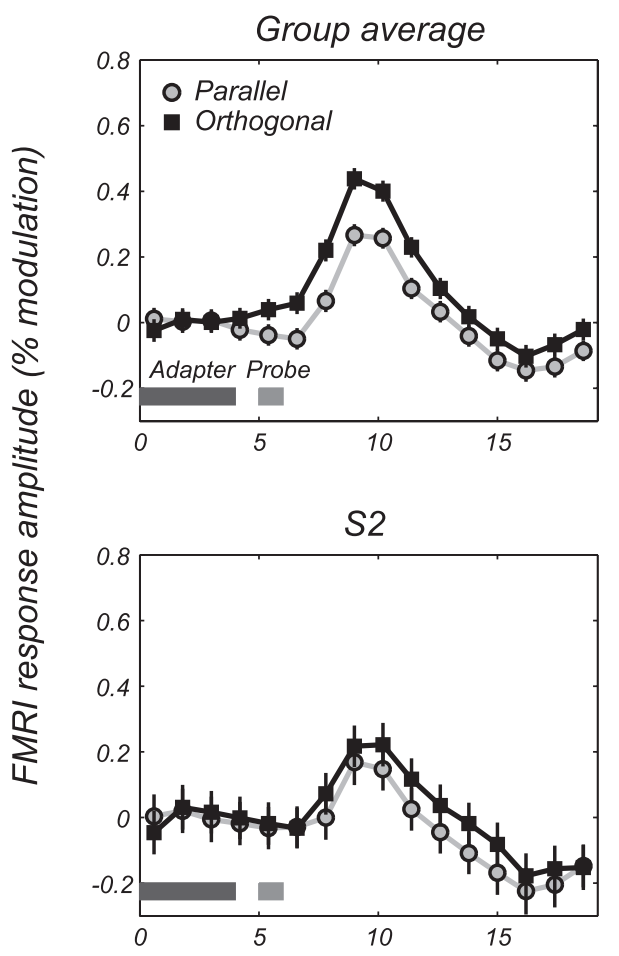

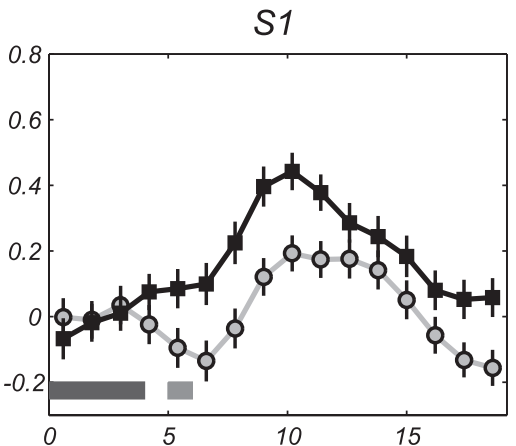

S3

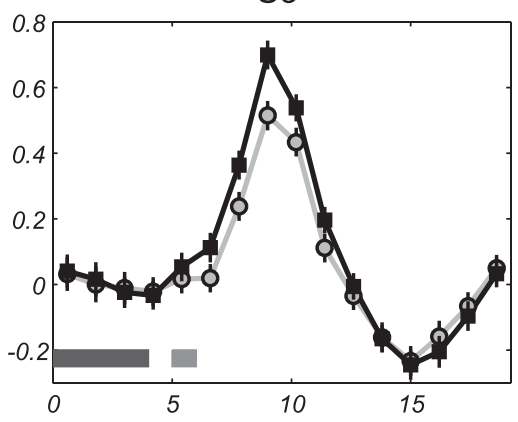

FIG. 6. Time-courses of V1 fMRI responses evoked by the probe stimuli in the LM:LM condition for individual subjects and averaged across subjects. Mean responses to adapter stimulus were subtracted from time-courses. Adapter and probe stimulus onset and duration are indicated by dark and light gray bars, respectively. In all subjects, measured response to orthogonal probe stimulus (black lines and square symbols) is stronger than response to parallel probe stimulus (gray lines and circle symbols), although difference is significant only in subjects $\mathrm{S} 1$ and $\mathrm{S} 3$.

Time (s)

for three lower-tier visual areas (V1, V2, and V3), two ventral higher-tier areas (hV4 and VO1), and a dorsal higher-tier area (LO1). The response amplitudes for all ROIs are summarized in Fig. 10A. Among the areas that exhibited significant adaptation, the response amplitudes were larger in early visual areas $(\mathrm{V} 1, \mathrm{~V} 2$, and V3) than in higher-tier visual areas (hV4, VO1, $\mathrm{V} 3 \mathrm{~A} / \mathrm{B}$, and LO1). In contrast, the adaptation indices were much the same across these areas (Fig. 11A), indicating that the relative amount of response adaptation in this condition did not vary between visual areas.
ADAPTATION TO SECOND-ORDER STIMULI (CONDITIONS CM:CM AND OM:OM). Areas that showed orientation-selective response adaptation to first-order gratings also showed orientation-selective response adaptation to second-order gratings (Table 1; Figs. 8, 9, 10, $B$ and $C$ ). Time-courses are plotted in Figs. 8 (condition $\mathrm{CM}: \mathrm{CM}$ ) and 9 (condition OM:OM). Response amplitudes were larger in early visual areas V1, V2, and V3 in conditions CM:CM and OM:OM than in the LM:LM condition, presumably reflecting the response to the carrier stimulus, which was not present in the LM:LM condition. In higher-tier

TABLE 1. Response amplitude difference (in units of \% FMRI signal change) between orthogonal and parallel trials, by condition for individual subjects and averaged across subjects for all visual area ROIs

\begin{tabular}{|c|c|c|c|c|c|c|c|c|c|c|c|}
\hline & V1 & V2 & V3 & $\mathrm{V} 3 \mathrm{~A} / \mathrm{B}$ & V7 & V5 & LO1 & LO2 & hV4 & VO1 & pLOC \\
\hline \multicolumn{12}{|l|}{ LM:LM } \\
\hline S1 & $0.46(0.002)$ & $0.32(0.002)$ & $0.31(0.005)$ & $0.36(0.014)$ & $0.22(0.035)$ & $0.15(0.11)$ & $0.23(0.013)$ & $0.19(0.024)$ & $0.27(0.035)$ & $0.16(0.083)$ & $0.15(0.12)$ \\
\hline S2 & $0.09(0.23)$ & $0.07(0.11)$ & $0.05(0.23)$ & $-0.01(0.53)$ & $-0.07(0.65)$ & $-0.10(0.86)$ & $0.03(0.27)$ & $-0.12(0.82)$ & $0.06(0.13)$ & $0.06(0.14)$ & $-0.31(0.89)$ \\
\hline S3 & $0.26(0.006)$ & $0.22(0.000)$ & $0.25(0.000)$ & $0.24(0.000)$ & $0.13(0.013)$ & $0.07(0.040)$ & $0.21(0.000)$ & $0.15(0.004)$ & $0.19(0.003)$ & $0.23(0.013)$ & $0.13(0.009)$ \\
\hline Group & $0.27(0.000)$ & $0.20(0.000)$ & $0.20(0.000)$ & $0.20(0.001)$ & $0.09(0.097)$ & $0.04(0.22)$ & $0.16(0.000)$ & $0.07(0.092)$ & $0.17(0.001)$ & $0.15(0.003)$ & $-0.01(0.55)$ \\
\hline \multicolumn{12}{|l|}{ CM:CM } \\
\hline S1 & $0.20(0.15)$ & $0.18(0.12)$ & $0.28(0.009)$ & $0.21(0.13)$ & $-0.17(0.94)$ & $-0.09(0.81)$ & $0.30(0.003)$ & $0.10(0.10)$ & $0.17(0.088)$ & $0.20(0.10)$ & $-0.01(0.52)$ \\
\hline S2 & $0.14(0.15)$ & $0.13(0.17)$ & $0.19(0.085)$ & $0.13(0.081)$ & $-0.05(0.63)$ & $-0.05(0.69)$ & $0.12(0.17)$ & $0.06(0.14)$ & $0.22(0.008)$ & $0.23(0.002)$ & $0.02(0.41)$ \\
\hline S3 & $0.10(0.15)$ & $0.18(0.000)$ & $0.27(0.000)$ & $0.25(0.000)$ & $0.07(0.054)$ & $-0.10(0.96)$ & $0.21(0.000)$ & $-0.12(0.93)$ & $0.14(0.030)$ & $0.25(0.000)$ & $-0.05(0.79)$ \\
\hline \multirow{2}{*}{\multicolumn{12}{|c|}{$0.25(0.000)-0.01(0.09)$}} \\
\hline & & & & & & & & & & & \\
\hline $\mathrm{S} 1$ & $0.20(0.16)$ & $0.20(0.064)$ & $0.11(0.19)$ & $0.03(0.42)$ & $0.06(0.27)$ & $0.08(0.15)$ & $0.08(0.19)$ & $0.07(0.18)$ & $0.14(0.12)$ & $0.05(0.34)$ & $-0.03(0.62)$ \\
\hline S2 & $0.04(0.41)$ & $0.09(0.23)$ & $0.21(0.041)$ & $0.06(0.039)$ & $0.12(0.16)$ & $0.04(0.28)$ & $0.02(0.37)$ & $-0.03(0.62)$ & $0.22(0.005)$ & $0.41(0.000)$ & $-0.01(0.51)$ \\
\hline S3 & $0.37(0.001)$ & $0.34(0.000)$ & $0.42(0.000)$ & $0.29(0.000)$ & $0.10(0.018)$ & $-0.02(0.70)$ & $0.20(0.080)$ & $-0.14(0.99)$ & $0.22(0.007)$ & $0.38(0.001)$ & $-0.17(0.99)$ \\
\hline Group & $0.20(0.015)$ & $0.21(0.001)$ & $0.25(0.000)$ & $0.13(0.015)$ & $0.09(0.043)$ & $0.03(0.17)$ & $0.10(0.087)$ & $-0.03(0.77)$ & $0.19(0.000)$ & $0.28(0.000)$ & $-0.07(0.86)$ \\
\hline \multicolumn{12}{|l|}{ LM:OM } \\
\hline $\mathrm{S} 1$ & $0.17(0.16)$ & $0.20(0.050)$ & $0.22(0.029)$ & $-0.38(0.99)$ & $-0.18(0.89)$ & $-0.10(0.75)$ & $0.07(0.12)$ & $-0.02(0.61)$ & $0.09(0.15)$ & $0.03(0.35)$ & $-0.20(0.76)$ \\
\hline S2 & $-0.36(0.97)$ & $-0.29(0.97)$ & $-0.19(0.92)$ & $-0.02(0.60)$ & $-0.23(0.98)$ & $-0.05(0.77)$ & $-0.07(0.81)$ & $0.09(0.18)$ & $-0.11(0.85)$ & $-0.11(0.94)$ & $0.03(0.36)$ \\
\hline S3 & $0.20(0.023)$ & $0.08(0.051)$ & $0.14(0.009)$ & $0.23(0.002)$ & $0.08(0.054)$ & $-0.02(0.74)$ & $0.12(0.021)$ & $0.12(0.020)$ & $0.14(0.024)$ & $0.26(0.007)$ & $0.01(0.43)$ \\
\hline Group & $0.00(0.49)$ & $-0.00(0.51)$ & $0.06(0.18)$ & $-0.06(0.79)$ & $-0.11(0.96)$ & $-0.05(0.85)$ & $0.04(0.14)$ & $0.06(0.060)$ & $0.04(0.22)$ & $0.06(0.12)$ & $-0.05(0.70)$ \\
\hline
\end{tabular}

$P$-values (numbers in parentheses) indicate probability of obtaining a smaller response to parallel trials than to orthogonal trials by chance (for individual subjects: one-tailed $t$-test, $\min \mathrm{df}=447$, $\max \mathrm{df}=508$; for group average: estimated from bootstrap generated distributions of the mean difference between orthogonal and parallel trials (averaged across the three subjects, see METHODS) Bold type indicates a significantly $(P<0.05)$ larger response to orthogonal than to parallel trials. 

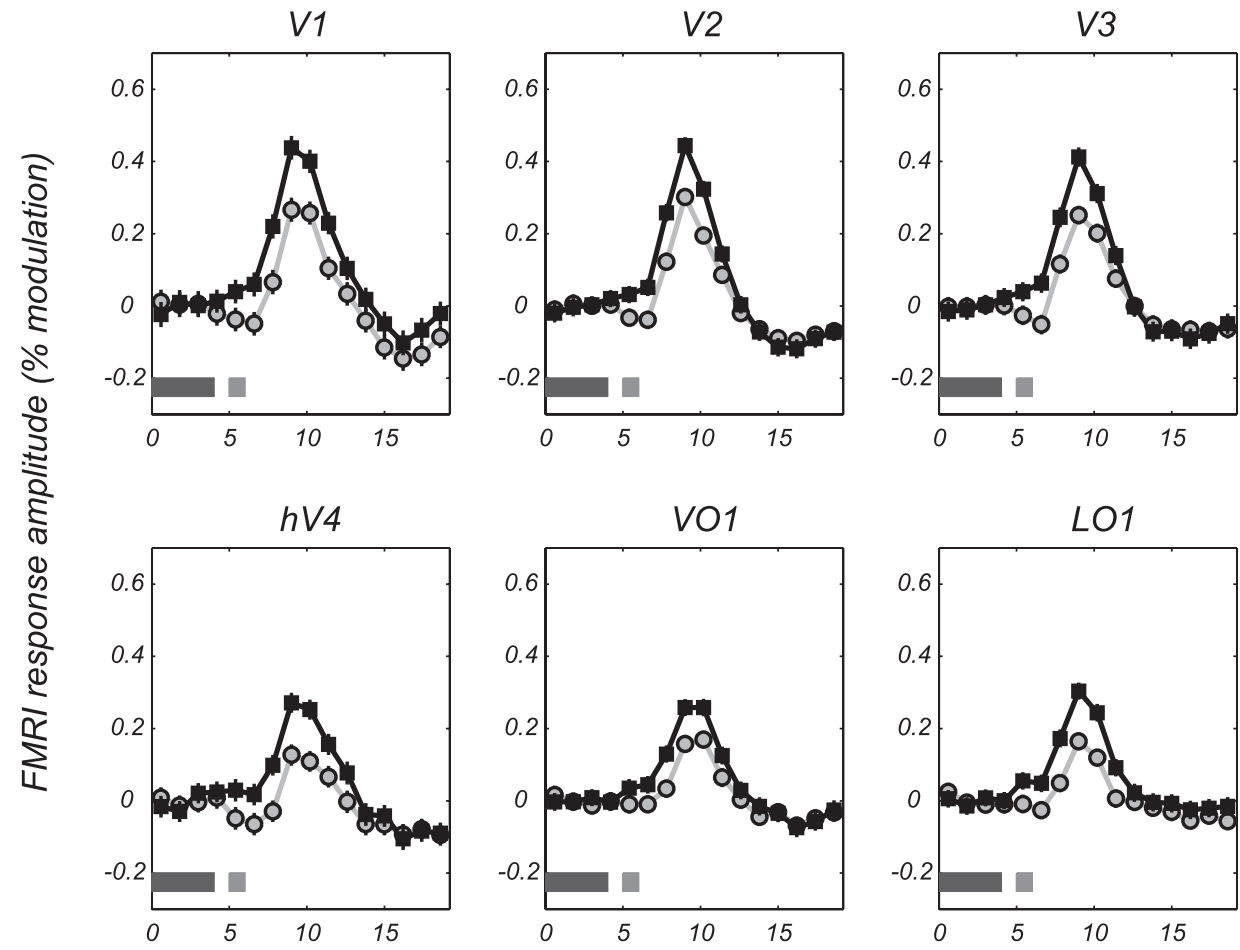

O Parallel

- Orthogonal
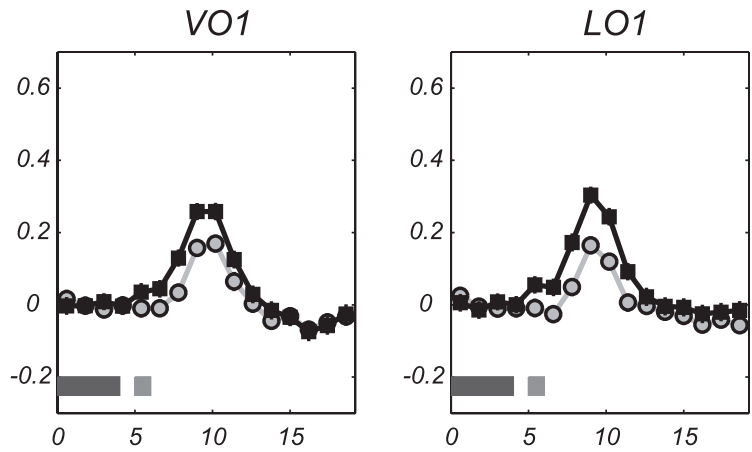

Time (s)

FIG. 7. Time-courses of fMRI responses, averaged across subjects, evoked by probe stimuli in the LM:LM condition for areas V1, V2, V3, hV4, VO1, and LO1. Same conventions as in Fig. 6.

areas, response amplitudes were similar for first- and secondorder stimuli. The magnitude of response adaptation, however, varied across visual areas, becoming progressively larger in

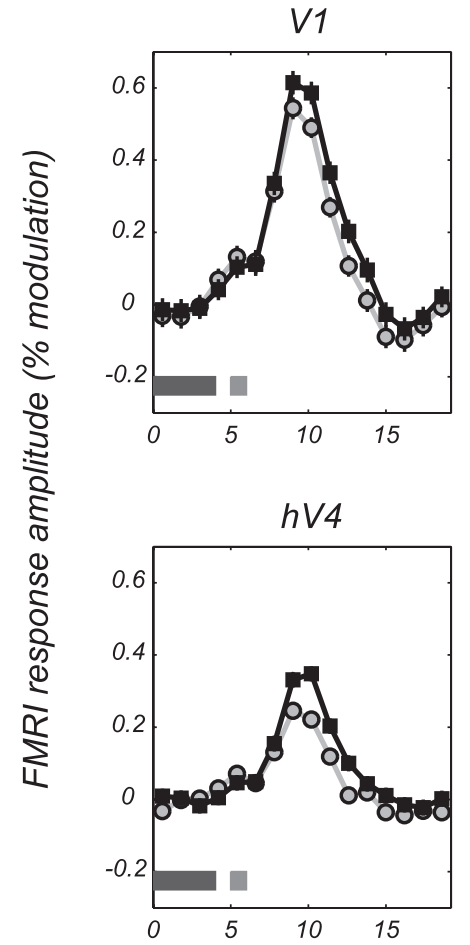

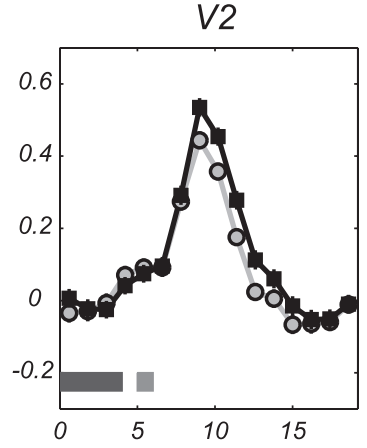
particularly striking in the adaptation indices (Fig. $11, B$ and $C)$, which were small $(\sim 0.1)$ in V1 but significantly larger

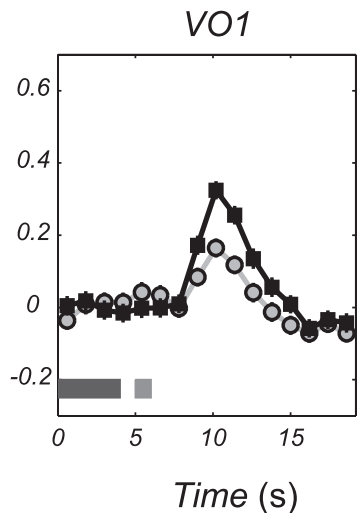

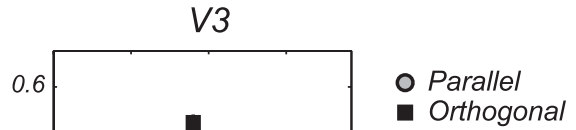

- Orthogonal

FIG. 8. Time-courses of fMRI responses, averaged across subjects, evoked by probe stimuli in the CM:CM condition for areas V1, V2, V3, hV4, VO1, and LO1. Same conventions as in Fig. 6. 

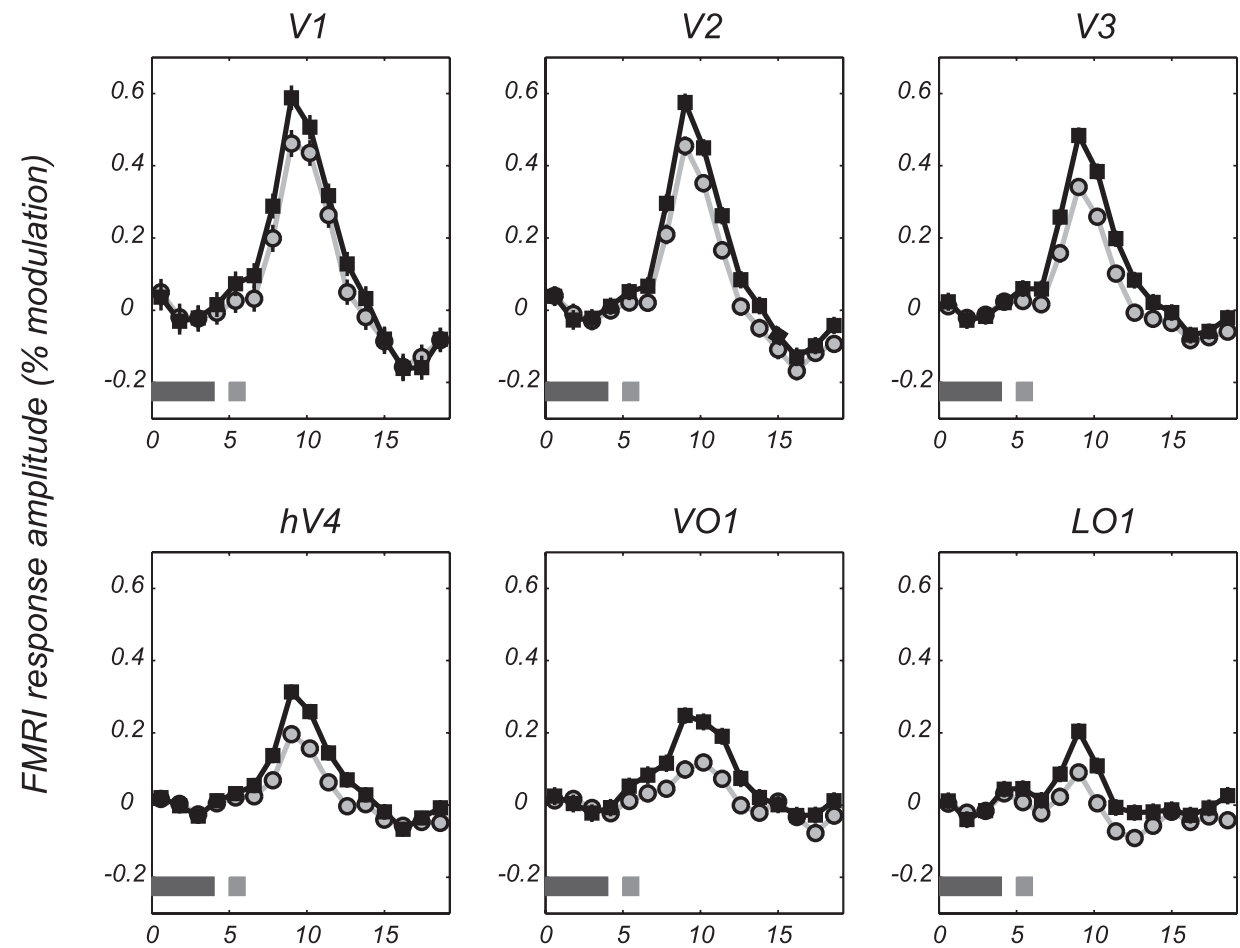

- Parallel

- Orthogonal
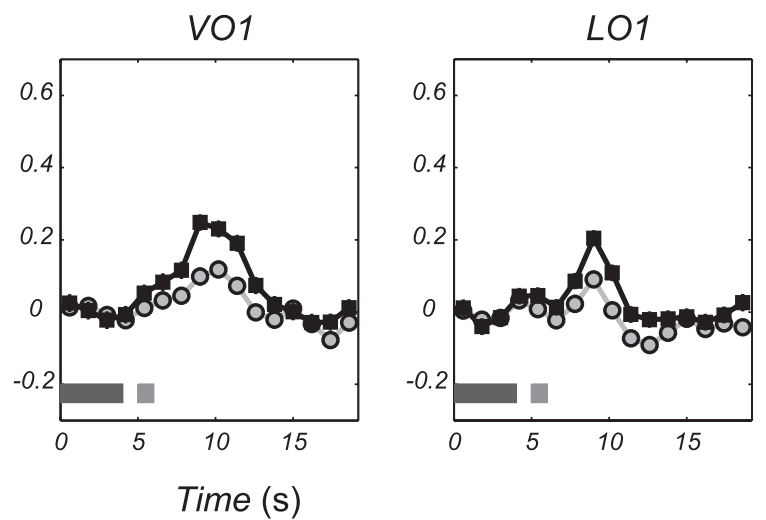

FIG. 9. Time-courses of fMRI responses, averaged across subjects, evoked by probe stimuli in the OM:OM condition for areas V1, V2, V3, hV4, VO1, and LO1. Same conventions as in Fig. 6.

$(\sim 0.3)$ in higher-tier areas, particularly VO1 (and in the CM:CM condition, also in areas V3A/B and LO1).

\section{Cross-modal adaptation between first- and second-order stimuli (condition LM:OM)}

The same probe stimuli were used in the unimodal condition $\mathrm{OM}: \mathrm{OM}$ and in the cross-modal condition LM:OM, and the pattern of response amplitudes was similar in the two conditions, with a relatively larger response in $\mathrm{V} 1-\mathrm{V} 3$ than in downstream extrastriate areas (Fig. 12A). Unlike condition OM:OM, however, there was no consistent evidence of orientation-selective adaptation in the cross-modal condition (Table 1; Fig. 12B): the response to the parallel stimulus did not differ significantly from the response to the orthogonal stimulus in any visual area except V7, where one of the subjects showed significant response enhancement to the adapted condition (opposite to the predicted effect of adaptation). There was greater variability within and between subjects in this condition than in the unimodal conditions, and the effects of adaptation were not consistent across subjects.

\section{Origin of extrastriate adaptation}

Because we found adaptation in multiple areas, a possible interpretation is that each of these areas contains neurons selective for first- or second-order stimulus orientation. However, a reduced response to the adapted stimulus in one area could also reflect a reduced input to the area caused by adaptation elsewhere. To interpret response reductions as evidence of selective adaptation of neurons within an area, it is necessary to show that the adaptation effect cannot be ac- counted for by a reduced input to the area. Failure to do so may lead to erroneous conclusions about the tuning and/or adaptation properties of the area. For example, single-unit recordings in macaque MT have suggested that direction-selective response adaptation in MT neurons is caused by adaptation of the V1 neurons projecting to MT, rather than reflecting properties of the MT neurons themselves (Kohn and Movshon 2004). A similar effect was recently shown in macaque V4 (Tolias et al. 2005). Given that V1 provides the major feed-forward input to extrastriate visual cortex, any response adaptation observed in this area will be propagated to downstream extrastriate visual areas. Therefore we would expect response adaptation in V1 to be associated with response adaptation in extrastriate visual areas, even if neurons in extrastriate areas do not themselves adapt to the stimuli (e.g., because they are not selective for stimulus orientation). Note that the adaptation effect in V1 could not have originated at an earlier, subcortical stage (i.e., in the LGN), because our experimental design ensured that only orientation-selective neurons would adapt differentially during orthogonal and parallel trials. Because LGN neurons are not selective for stimulus orientation, any adaptation in these neurons (Solomon et al. 2004) would have been equal for both trial types.

V1 response adaptation accounted for most of the observed extrastriate adaptation to first-order stimuli: in condition LM: LM, the adaptation index was approximately constant across the visual areas that responded to the stimuli (Fig. 11A). This result implied that neurons responding to first-order orientation in extrastriate visual areas such as V2 and V3 did not themselves adapt strongly to the simple grating stimuli used in our experiments. Rather, we suggest that most of the adaptation in 
A

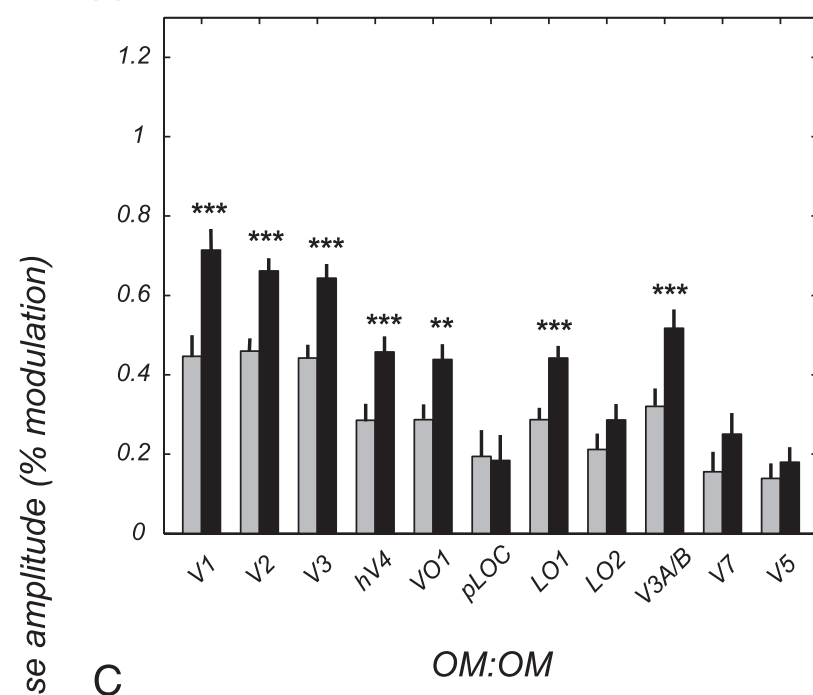

B

$\frac{5}{\text { की }}$
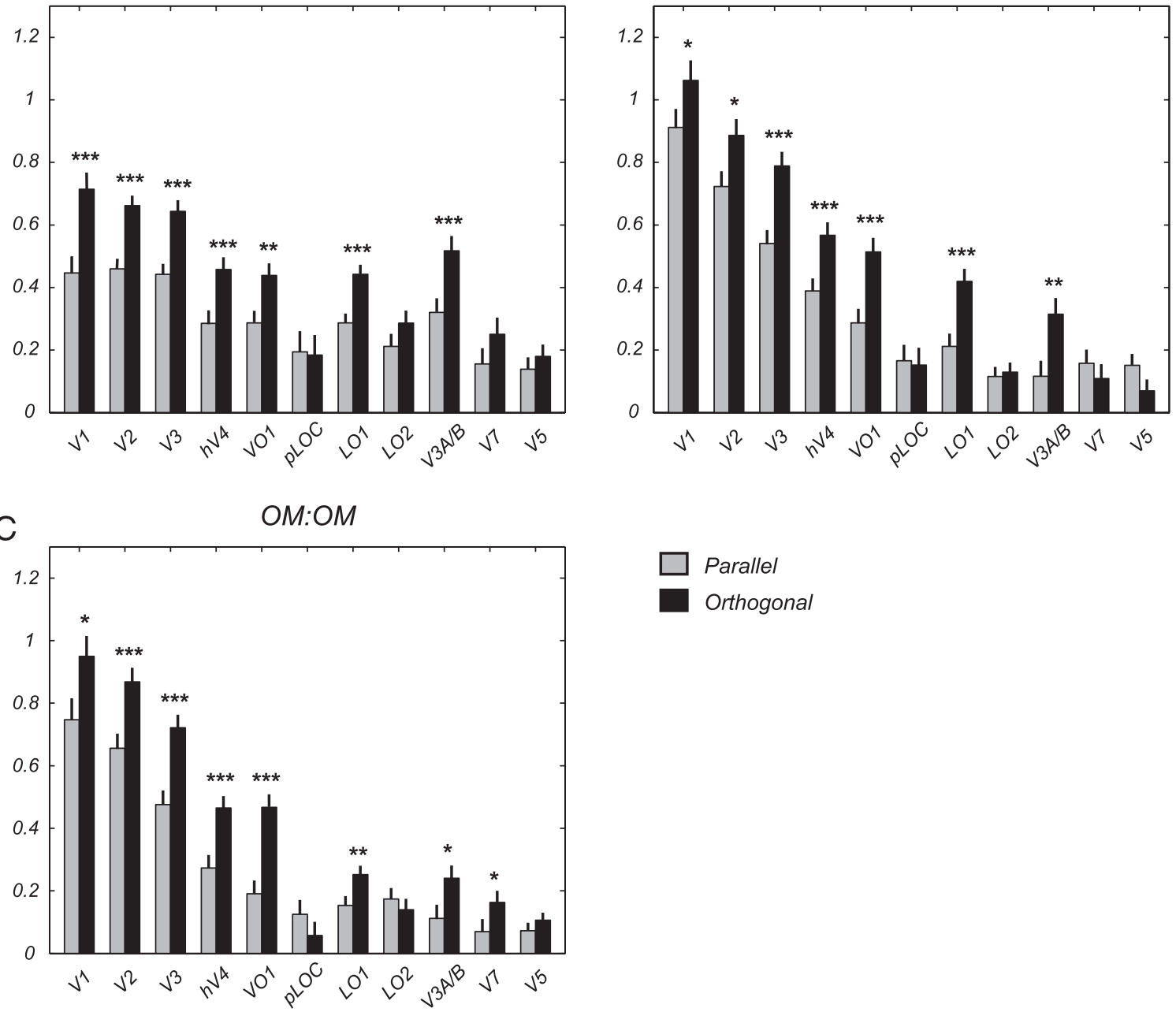

Orthogonal

\section{Visual area}

FIG. 10. Response amplitudes, averaged across subjects, for all visual area ROIs in the unimodal conditions. Response amplitudes to parallel probe stimuli are shown in light gray; responses to orthogonal probe stimuli are shown in black. Asterisks indicate a statistically significant difference between group average responses to orthogonal and parallel probes $\left(* P<0.05\right.$; $* * P<0.01$; ${ }^{*} * P<0.001$; Table 1). A: condition LM:LM. $B$ : condition CM:CM. $C$ : condition OM:OM. Error bars represent SE of the 3 subjects.

this condition originated in V1, with little additional adaptation in downstream extrastriate areas. One possible interpretation of this result is that neurons in extrastriate areas are selective for stimulus orientation, but do not adapt as strongly as V1 neurons. A more likely reason for the absence of additional adaptation in extrastriate visual areas is that neurons in these areas are selective for more complex features than the orientation of luminance-defined gratings (Gallant et al. 1993, 1996; Ghose and Ts'o 1997; Hegde and Van Essen 2000, 2003; Ito and Komatsu 2004; Lui et al. 2005; Mahon and De Valois 2001; Marcar et al. 2000; Pasupathy and Connor 1999, 2001; Thomas et al. 2002). These neurons are unlikely to show strong adaptation to nonpreferred stimuli, such as the sinusoidal luminance gratings used in condition LM:LM.

In contrast, the adaptation indices in both second-order conditions (CM:CM and OM:OM) were larger in several extrastriate visual areas than in V1, although the difference was statistically significant only in VO1 in both conditions (and also in V3A/B and LO1 in the CM:CM condition; Fig. $11, B$ and $C$ ). This result indicated that the response adap- tation in these areas could not be fully accounted for by adaptation in V1. We interpret this as evidence that the response adaptation in extrastriate areas was at least in part caused by local adaptation within these areas, implying that they contained populations of neurons that computed (and adapted to) second-order stimulus orientation. An alternative explanation is that the activity in one or more of the extrastriate areas was driven by the output of the subpopulation of V1 neurons that exhibited the strongest adaptation, with no additional adaptation beyond V1. It is possible that the orientation of second-order patterns was computed in only a single extrastriate area (e.g., LO1), and that the adaptation observed in other areas (e.g., VO1) was simply propagated from this one area, in the same way that adaptation to firstorder orientation in V1 was propagated to extrastriate areas. We cannot determine from our current data whether the adaptation we observed in extrastriate visual areas was due to selective adaptation in another upstream extrastriate area or whether it reflected independent stages of processing in multiple extrastriate visual areas. 

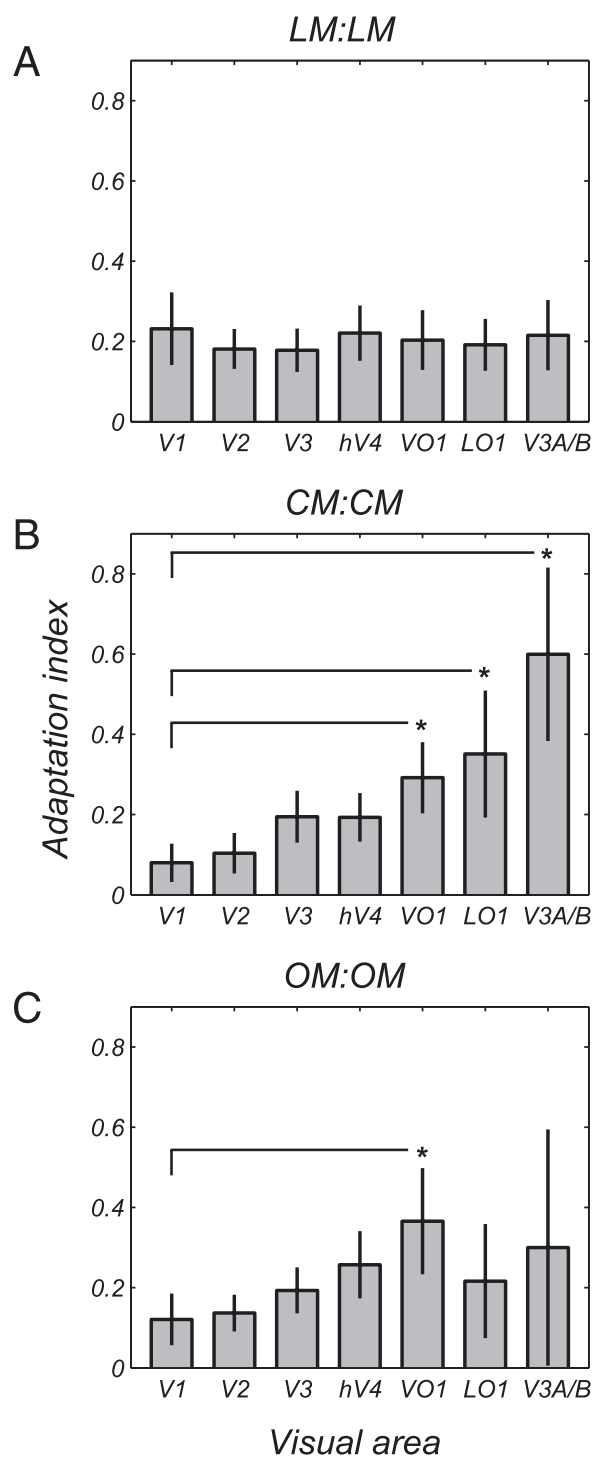

FIG. 11. Adaptation indices, averaged across subjects, for the seven visual ROIs showing the strongest responses across all unimodal conditions. A: condition LM:LM. B: condition CM:CM. $C$ : condition OM:OM. For each extrastriate area, asterisks indicate a significantly larger adaptation index in that area than in V1 $(P<0.05)$. Error bars represent $84 \%$ confidence interval for mean of the 3 subjects, estimated from bootstrap-generated distributions of means in individual subjects.

Differences in distribution of first- and second-order response adaptation across visual areas

The pattern of response amplitudes and adaptation amplitudes for the two second-order conditions $\mathrm{CM}: \mathrm{CM}$ and $\mathrm{OM}: \mathrm{OM}$ were more similar to one another than to the other conditions (Figs. 10 and 13). This result is most apparent in a scatter plot of the adaptation indices across the different conditions (Fig. 13). In these plots, a similar distribution of adaptation across visual areas in two conditions would yield data points lying along the diagonal. The adaptation indices from conditions CM:CM and OM:OM clustered along the diagonal (albeit with some variability), indicating a positive correlation between the two conditions (Fig. 13A). Such a correlation was less evident when the adaptation indices for either second-order condition were plotted against the adaptation indices of the first-order condition LM:LM (Fig. 13, $B$ and
C). Furthermore, unlike the first-order condition, the adaptation indices of the second-order conditions showed a clear dissociation between lower-tier and higher-tier visual areas: less strongly adapting early visual areas V1, V2, and V3 (plotted in shades of blue) formed a cluster in the lower left-hand corner of Fig. 13A, whereas the more strongly adapting higher-tier areas hV4, V3A/B, LO1, and VO1 (in shades of red, yellow, and green) were displaced upward and to the right along the diagonal. In the first-order condition, adaptation indices were relatively constant across visual areas, and as a consequence, lower-tier and higher-tier areas did not form distinct clusters in the plots that include the first-order condition (Fig. 13, $B$ and $C$ ). If first- and second-order patterns were detected by a single common mechanism, they would have evoked similar patterns of response adaptation across visual areas. Our finding that the distribution of response adaptation differed between first- and second-order conditions therefore adds to the body of evidence suggesting that first- and second-order pattern perception are mediated by different neuronal mechanisms. Furthermore, the fact that the pattern of response adaptation to second-order stimuli did not depend strongly on second-order stimulus type-i.e., whether the stimuli were defined by modulations of contrast (CM stimuli) or orientation (OM stimuli) - implies that either a single population of neurons mediates detection of both types of second-order patterns or that the two types of second-order patterns are detected by different populations of neurons which are similarly distributed across visual areas.

\section{I S C U S S I O N}

Our results indicate that neurons selective for second-order orientation are found in most retinotopically organized visual areas. Furthermore, because the amplitude of response adaptation to second-order patterns was similar to that found for first-order patterns, neurons selective for second-order orientation appear to be as abundant as neurons selective for first-order orientation (in human visual cortex). These findings are consistent with single-unit recordings from cats and monkeys that indicate that neurons selective for second-order stimuli are not uncommon, at least in extrastriate visual areas (Leventhal et al. 1998; Mareschal and Baker 1998a,b, 1999; O'Keefe and Movshon 1998; Zhou and Baker 1993, 1994, 1996).

In agreement with previous neuroimaging studies of secondorder motion (Dupont et al. 2003; Nishida et al. 2003; Seiffert et al. 2003), we found little evidence for the existence of spatially segregated pathways for first- and second-order pattern processing. On the other hand, there is considerable psychophysical evidence that first- and second-order visual processing are mediated by different mechanisms, and our results are consistent with the existence of separate mechanisms for first- and second-order pattern perception. First, orientation-selective adaptation to first-order stimuli was roughly constant across visual areas, whereas selective adaptation to second-order stimuli was significantly larger in several extrastriate visual areas. Second, we failed to find consistent cross-adaptation in the LM:OM condition.

\section{Orientation-selective adaptation in VI}

Consistent with predictions from single-unit physiology, we found evidence for orientation-selective adaptation to first- 
A

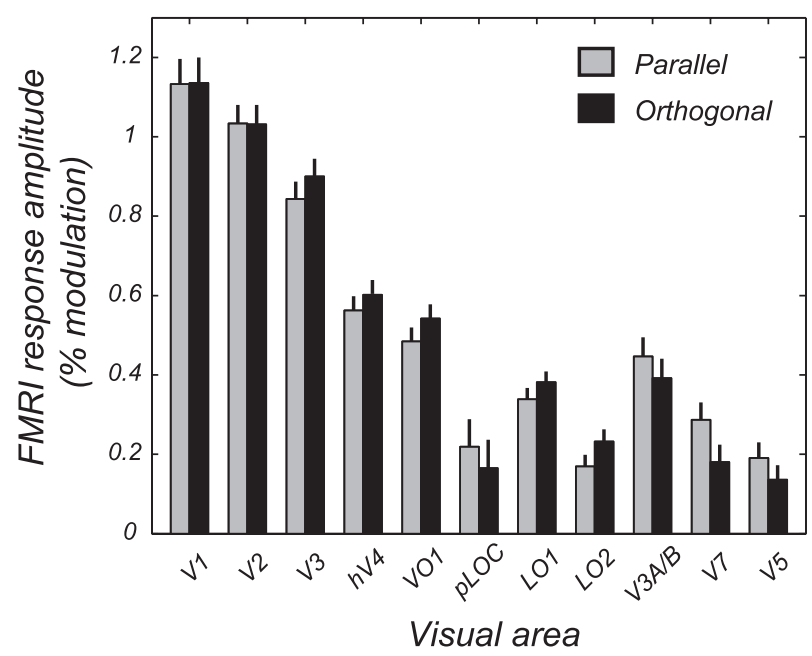

B

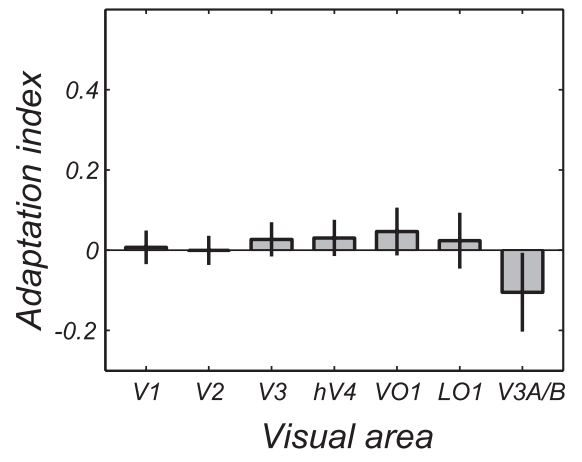

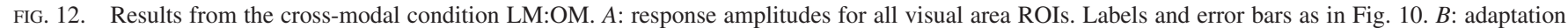
indices for the same visual area ROIs shown in Fig. 11. Labels and error bars as in Fig. 11.

order stimuli (condition LM:LM) in area V1. This correspondence between the known tuning properties of single neurons and the tuning properties inferred from measurements of population activity provides important validation of the use of response adaptation to study neuronal response selectivity with fMRI. Surprisingly, unambiguously showing orientation-selec-

A

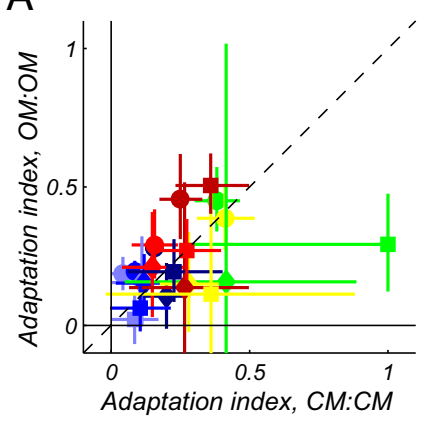

$\mathrm{C}$

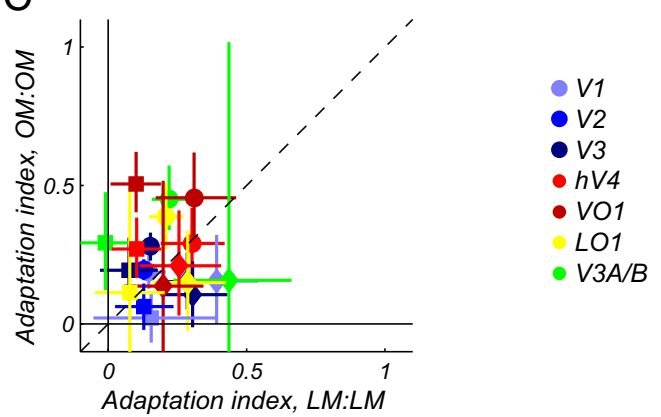

tive adaptation in human V1 by fMRI has not been straightforward. In the first study that used adaptation to measure orientation selectivity, Tootell et al. (1998b) reported orientation-selective transient responses in V1 after changing the orientation of a high-contrast grating after prolonged adaptation. However, because attention was not controlled, these transient responses could in principle have reflected an attentional signal elicited by the change in the stimulus rather than selective adaptation to stimulus orientation. Kourtzi et al. (2003) used both prolonged adaptation and a rapid adaptation protocol and found transient responses in V1 associated with changes in random patterns of Gabor patches or line segments, both in anesthetized monkeys and in human subjects (with and without controlling attention). Such transients are consistent with orientation-selective adaptation. In a more direct attempt at measuring orientation-selective adaptation, Boynton and Finney (2003) used a rapid adaptation protocol, in which pairs of parallel or orthogonal high-contrast gratings were presented in succession (as opposed to the prolonged preadaptation and top-up adaptation used in our study), with attention tightly controlled by a highly demanding task at fixation. Boynton and Finney found no evidence of orientation-selective adaptation in V1 and suggested a number of reasons for this result. For example, the use of a rapid adaptation protocol may have failed to induce sufficiently profound neuronal adaptation in V1. Alternatively, the fact that the spatial frequency of the stimuli used was much lower than optimal for V1 may have made them ineffective as adapting stimuli. A third possibility is that the high contrast of the stimuli may have led to neuronal response saturation, masking the adaptation effect. In a previous study, in which we used high-contrast stimuli both for inducing and measuring adaptation and a block design as opposed to the event-related design of this study, we also failed to find orientation-selective adaptation in V1 (Larsson et al. 2003). In the present study, we chose a high contrast for the adapter to evoke strong adaptation but an intermediate contrast for the probe. The probe contrast was chosen to be both high enough to give rise to a robust fMRI response and sufficiently low to avoid neuronal response saturation, as determined from single-unit measurements of adaptation (Carandini et al. 1997,
FG. 13. Scatter plots of adaptation indices across conditions. Error represent the $84 \%$ confidence interval for the mean of adaptation indices showing the strongest responses. A: adaptation indices in condition OM:OM vs. condition CM:CM (2nd-order vs. 2nd-order). Adaptation indices for lowertier visual areas (V1, V2, and V3) form a cluster in the bottom left corner; indices for higher tier areas are displaced upward and to the right along the diagonal. $B$ : adaptation indices in condition CM:CM vs. condition LM:LM (2nd-order vs. 1st-order). $C$ : adaptation indices in condition OM:OM vs. condition LM:LM (2nd-order vs. 1st-order). In $B$ and $C$, lower- and higher-tier visual areas do not form distinct clusters along the LM:LM axis. 
1998; Sclar et al. 1989). The most compelling evidence so far for orientation-selective adaptation in human V1 comes from Engel (2005), who also used a combination of high-contrast adapter and low-contrast probe stimuli, as well as a task to control attention. In contrast with our study, the task used by Engel required subjects to direct attention to the stimuli. Our results confirm and extend those of Engel; specifically, because we actively diverted attention from the stimuli, our study is the first to show that response adaptation in early visual areas does not require attention, in contrast with higher-order areas such as the pLOC (Eger et al. 2004). This result agrees with single-unit measurements of V1 response adaptation, most of which have been performed in the absence of attention (i.e., under anesthesia), as well as psychophysical studies that show adaptation to luminance gratings in the absence of attention (Festman and Ahissar 2004; He and MacLeod 2001).

\section{Adaptation versus change detection}

An alternative possible interpretation of our results is that the stronger response to the (comparatively) infrequently presented orthogonal (nonadapted) stimulus orientation could be caused by a change or novelty detection signal. This effect cannot explain our data for two reasons. First, because of the severe attentional demands of the foveal task, subjects were mostly unaware of the orientation of the probe stimulus; one would presume a change signal would be associated with a behavioral detection of stimulus changes. Second, a change signal would be identical for different types of visual stimuli, and this is hard to reconcile with our finding of systematic differences between the pattern of first- and second-order response adaptation across visual areas (as well as the absence of adaptation in the cross-modal condition).

\section{Early nonlinearities}

A potential confound in all studies of second-order visual processing is the presence of first-order artifacts in the secondorder stimuli. While we made every effort to ensure that our display hardware did not introduce such artifacts by careful calibration of the monitor output, it is difficult to guarantee their complete absence. However, given the potential error in our calibrations and the range of stimulus contrasts used, these artifacts would have been very small ( $<1 \%$ luminance contrast in the CM stimuli and much smaller still in the OM stimuli), well below the psychophysical detection thresholds for firstorder stimuli with corresponding spatial frequency and eccentricity (Fig. 5). A potentially more serious issue is that early nonlinearities in the neuronal transduction of physically pure second-order stimuli could in principle introduce first-order artifacts detectable by first-order mechanisms. Although we cannot rule out the possibility that such nonlinearites exist, they cannot account for the adaptation effects we observe. As Baker and Mareschal (2001) and many others have noted, any early nonlinearities must be very small to accommodate the large body of evidence for the approximately linear properties of V1 neurons, much smaller than would be required for them to mediate second-order pattern or motion perception. Given that the response and adaptation magnitudes we observed in the second-order conditions were of similar magnitude as those in the first-order condition, early nonlinearities are unlikely to be the main source of these effects.

\section{Comparison with single-unit studies}

While many of our findings agree with previous single-unit studies and extend these studies by measuring selectivity for second-order patterns in extrastriate areas other than V2 and V5/MT, there are some noteworthy differences. First, we did not find a strong dissociation between V1 and V2 in terms of second-order orientation selectivity. Although single units selective for second-order patterns have been found in V1, several studies report that they are much less frequent in V1 than in V2, and so we might have expected to find a greater difference between the adaptation indices in these areas than we observed. In this respect, our results are similar to those of Nishida et al. (2003), who found direction-selective adaptation both to first- and second-order motion in V1 (as well as in extrastriate visual areas). Second, we failed to find consistent evidence for cross-modal adaptation in any visual area, whereas a number of single-unit studies (Albright 1992; Leventhal et al. 1998; Mareschal and Baker 1999) have found neurons that respond both to first- and second-order patterns in a cue-invariant manner.

These discrepancies could reflect genuine species differences. Humans and macaques are separated by $\sim 30$ million years of divergent evolution, and the evolutionary distance between cats (the species used in the studies by Baker and coworkers; Mareschal and Baker 1998a,b; Zhou and Baker $1993,1994,1996)$ and humans is greater still. It is unlikely that the organization and neuronal response properties of the visual cortex of humans and other mammals, even Old World primates, are exactly homologous.

The discrepancies between our results and those reported from electrophysiology studies could instead be caused by differences in experimental methods and design. As pointed out by Baker and Mareschal (2001), the failure to find substantial numbers of neurons tuned to second-order patterns in V1 in single-unit studies could be a result of the methods used to isolate neurons for recording. In most single-unit studies, neurons are isolated using first-order stimuli (such as luminance gratings or bar stimuli). Neurons selective for secondorder but not first-order orientation would not respond well to such stimuli and would therefore be excluded by this procedure, even if they were relatively common in V1. By definition, the only second-order-selective neurons isolated by this procedure are those that also respond to first-order stimuli, and the distinction between V1 and V2 observed in these studies therefore only applies to cue-invariant neurons, as opposed to neurons tuned only to second-order stimuli. In the present experiment on the other hand, both neurons tuned only to second-order patterns and cue-invariant neurons would have contributed to the measured response adaptation. The fact that we did not observe consistent cross-modal adaptation (reflecting the responses of cue-invariant neurons) in condition LM:OM suggests that most of the response adaptation we found in the corresponding unimodal condition OM:OM was elicited by neurons selective only for second-order patterns, but not for first-order patterns.

Another important methodological difference between our study and single-unit studies is the measure of neural activity used. Whereas single-unit recordings measure spike rates, the BOLD signal measured by fMRI is believed to reflect not only the spiking output from a region of cortical tissue, but also the 
synaptic input and local processing within that region of tissue (Logothetis et al. 2001). V1 receives synaptic feedback connections from extrastriate cortex (e.g., Felleman and Van Essen 1991; Rockland and Pandya 1979), and it is entirely possible that, even in the absence of attention, some proportion of the measured fMRI response in V1 reflects this feedback input. If the feedback connections to V1 come from extrastriate neurons selective for second-order orientation, a proportion of the measured signal induced by the synaptic input from these connections will be orientation-selective and masquerade as V1 orientation selectivity for second-order patterns. Such selectively tuned feedback need not be visible in the spiking output properties of most V1 neurons, if the feedback projections serve to modulate rather than drive the activity of V1 neurons. Because feedback projections from extrastriate cortical areas to V1 tend to be diffuse and widespread (Salin and Bullier 1995), a feedback signal that leads to spiking activity in only a few V1 neurons could still elicit a measurable hemodynamic response. Unfortunately from our data, we cannot distinguish what part of the fMRI signal is caused by feedback synaptic input and what part reflects spiking output.

Finally, our study differs from single-unit studies in that we used selective adaptation to infer neuronal response properties rather than directly measuring the activity of individual neurons. This technique relies on the assumption that neurons exhibit selective response adaptation (i.e., reduced firing rates) to prolonged presentations of their optimal stimuli. While it is well established that V1 neurons selective for first-order orientation adapt to visual stimuli in this manner (Carandini et al. 1998; Sclar et al. 1989), little is known about the adaptation properties of neurons selective for second-order orientation. If the cue-invariant neurons (neurons selective for both 1st- and 2nd-order stimuli) found in single-unit studies do not adapt as strongly as neurons selective for first-order orientation, it would explain why we did not observe consistent orientationselective adaptation in the cross-modal condition (LM:OM). This interpretation is supported by our finding of little or no psychophysical adaptation in the cross-modal condition, in contrast with the results for the unimodal conditions (Fig. 5). Furthermore, spatial attention is known to enhance behavioral adaptation (Chaudhuri 1990; Festman and Ahissar 2004; Montaser-Kouhsari and Rajimehr 2004; Rezec et al. 2004; Sohn et al. 2004; Spivey and Spirn 2000). There is evidence that higher-order cortical areas (such as the LOC) only exhibit response adaptation (measured by fMRI) when attention is directed to the stimuli (Eger et al. 2004; Moradi et al. 2005; Yi and Chun 2005). Our failure to find cross-modal response adaptation could therefore also be a result of our experimental design that required subjects to divert their attention from the stimuli. Yet another possibility is that the number of cueinvariant neurons is simply not large enough to evoke a robust response. Given that the magnitude of response adaptation in $\mathrm{V} 1$ even in the first-order condition was modest, despite the pronounced orientation selectivity of a majority of V1 neurons, the response adaptation evoked by cue-invariant neurons could be too small to detect reliably with the fMRI methods we used.

Single-unit measurements of second-order orientation selectivity in macaque visual cortex have thus far been restricted to areas V1 and V2. No directly comparable recordings have been made in areas V3, V4, or posterior IT (a possible homolog of VO1). Assuming that human and monkey visual cortex have a similar organization, our results suggest that single-unit recordings in these higher-order areas are likely to find a substantial proportion of neurons selective for the orientation of secondorder patterns. Our finding that orientation-selective response adaptation to second-order stimuli can be reliably measured in early visual areas suggests that it may be fruitful to search for neurons that are selective for second-order orientation, but not selective for first-order orientation. Such measurements are also essential to resolve to what extent our measurements of fMRI response adaptation in early visual areas reflect processing intrinsic to those cortical areas as opposed to modulatory feedback from higher visual cortical areas.

\section{Separate mechanisms for first- and second-order pattern perception?}

The results of our study suggest that neurons selective for first- and second-order orientation constitute two distinct populations but that both types of neurons are found in most visual areas. It is obviously possible that first- and second-order selective neurons are spatially segregated at a finer (e.g., columnar) spatial scale, akin to the (relative) segregation of neurons tuned for orientation and color in V1 in blobs and pinwheels; such a fine-scale organization would not be resolvable by our current fMRI methods. The adaptation index, an indicator of the relative abundance of orientation-selective neurons, differed for first- and second-order stimuli in several visual areas. Whereas the adaptation index was of roughly the same magnitude in V1 and extrastriate visual areas for firstorder stimuli, for second-order stimuli, the adaptation index was significantly larger in several extrastriate areas than in V1. The response adaptation to first-order stimuli we observed in extrastriate areas could be accounted for by adaptation in V1 propagating to downstream areas. In contrast, for second-order stimuli, adaptation in V1 could account only for a portion of the adaptation seen in downstream extrastriate visual areas, particularly in area VO1. This indicates that neurons selective for second-order stimulus orientation become more common as one descends the ventral processing stream. Although there are no directly comparable single-unit data from monkey V3 or $\mathrm{V} 4$, recordings from monkey $\mathrm{V} 1$ and $\mathrm{V} 2$ also show that neurons responding to second-order stimuli are more abundant in extrastriate cortex than in V1 (e.g., Albright 1992; Chaudhuri and Albright 1997; Leventhal et al. 1998; von der Heydt and Peterhans 1989; von der Heydt et al. 1984).

\section{Implications for models of second-order mechanisms}

The adaptation protocol used in these experiments was designed to selectively identify response adaptation in neurons selective for second-order orientation, corresponding to the second stage of filtering in a FRF mechanism. We designed our experiment in such a way that carrier adaptation would have been constant across trial types, and hence the observed adaptation in the CM:CM and OM:OM conditions does not reveal anything about the properties of the first-stage filters.

Our results imply that relative numbers of second-stage neurons are larger in higher-tier extrastriate visual areas. However, the fact that we found adaptation to second-order stimuli in lower-tier visual areas (V1 and V2) suggests there are second-stage neurons also at early levels of cortical processing 
(assuming the adaptation in these areas reflects reduced firing rates, not just subthreshold feedback from higher-tier areas). It remains to be seen whether the second-stage neurons in lowerand higher-tier visual areas differ in terms of stimulus selectivity or response properties. Although FRF models do not explicitly predict the existence of multiple populations of second-stage neurons, psychophysical evidence for different second-order spatial frequency channels (Landy and Oruç 2002) is consistent with the existence of multiple sets of second-stage filters tuned to different spatial frequencies. The second-stage neurons in lower- and higher-tier visual areas could thus correspond to the different spatial frequency channels observed psychophysically. This would predict that the spatial frequency tuning of the second-stage neurons should differ across visual areas. Because we only used a single second-order spatial modulation frequency, we cannot test this prediction based on the data from this experiment. Alternatively, the adaptation in extrastriate areas could reflect the activity of a third filtering stage, mediated by neurons that sum the output of the second-stage filters in lower-tier areas. Again, additional experiments will be required to distinguish between these hypotheses.

An open question is whether different types of second-order stimuli (e.g., patterns modulated by contrast or orientation) are analyzed by a single mechanism or whether there are multiple second-order mechanisms. Using a masking paradigm, Kingdom et al. (2003) found that contrast- and orientation-modulated second-order stimuli are unlikely to be processed by a single mechanism, although their results were consistent with (different) FRF processes underlying detection of both types of stimuli. We found quite similar response and adaptation patterns for both contrast- and orientation-modulated stimuli, despite differences in carrier structure (isotropic vs. oriented) and overall contrast. If different mechanisms mediate detection of these different types of second-order patterns, our results imply that these mechanisms are not spatially segregated by visual area. A more direct test of whether a single or multiple mechanisms underlie different second-order pattern perception is to use fMRI to measure the strength and distribution of cross-adaptation between different types of second-order stimuli, and we plan to do this in future studies.

\section{Second-order selectivity in dorsal and ventral stream areas}

Our results are consistent with those of Kastner et al. (2000), who found that orientation-defined texture boundaries evoked stronger responses than uniform textures in V3A and two ventral areas they refer to as V4 and TEO, but extend these results by showing orientation selectivity for second-order patterns in these areas. [The ventral areas V4 and TEO presumably correspond to areas hV4 and VO1 in our study, although an exact correspondence is difficult to establish because Kastner et al. (2000) did not define visual areas on a flattened surface representation.] Furthermore, unlike Kastner et al. (2000), we found substantial selectivity for second-order pattern orientation in V3 and-although less consistentlyalso in V1 and V2. This difference in results might be caused by the higher sensitivity of our scanner hardware ( 3 vs. $1.5 \mathrm{~T}$, 4-channel phased-array surface coil vs. head coil), but could also reflect the fact that we used adaptation rather than subtraction to identify selective responses to second-order pat- terns. We did not observe selectivity for second-order orientation in pLOC, a region generally believed to be selective for more complex shapes. The new retinotopic area we have designated $\mathrm{LO} 2$ does not seem to be involved in processing of second-order patterns, as it showed little evidence of orientation-selective adaptation for these stimuli. In this regard, it was more similar to either its anterior (V5/MT) or ventral (pLOC) neighbors than to its posterior neighbor, LO1. Although we have not yet characterized the response properties of LO1 and LO2 for other stimulus categories, these results at least suggest that LO1 and LO2 differ in their function and stimulus selectivity. Our results show that second-order patterns are processed both in dorsal (V3A/B) and ventral (hV4, VO1) areas, although only in ventral area VO1 was there consistent evidence of adaptation to second-order patterns that could not be accounted for by adaptation in V1. While these results imply that processing of second-order patterns is not restricted to a single visual area or visual pathway, they suggest that the selectivity for such stimuli may be greater in ventral stream areas than in dorsal stream areas.

\section{A C K N OW LED G M EN T S}

The authors thank D. Schluppeck for helpful discussion and comments.

\section{G R A N T S}

This study was supported by National Eye Institute Grant RO1-EY-16165 and RO1-EY-11794 and by a grant from the Seaver Foundation.

\section{REFERENCES}

Aguirre GK, Zarahn E, and D'Esposito M. The variability of human, BOLD hemodynamic responses. Neuroimage 8: 360-369, 1998.

Albright TD. Form-cue invariant motion processing in primate visual cortex. Science 255: 1141-1143, 1992.

Arsenault AS, Wilkinson F, and Kingdom FA. Modulation frequency and orientation tuning of second-order texture mechanisms. J Opt Soc Am A 16: 427-435, 1999.

Backus BT, Fleet DJ, Parker AJ, and Heeger DJ. Human cortical activity correlates with stereoscopic depth perception. J Neurophysiol 86: 20542068, 2001.

Baker CL Jr and Mareschal I. Processing of second-order stimuli in the visual cortex. Prog Brain Res 134: 171-191, 2001.

Biswal B, Hudetz AG, Yetkin FZ, Haughton VM, and Hyde JS. Hypercapnia reversibly suppresses low-frequency fluctuations in the human motor cortex during rest using echo-planar MRI. J Cereb Blood Flow Metab 17: 301-308, 1997a.

Biswal B, Van Kylen J, and Hyde JS. Simultaneous assessment of flow and BOLD signals in resting-state functional connectivity maps. NMR Biomed 10: 165-170, 1997b.

Biswal B, Yetkin FZ, Haughton VM, and Hyde JS. Functional connectivity in the motor cortex of resting human brain using echo-planar MRI. Magn Reson Med 34: 537-541, 1995.

Boynton GM and Finney EM. Orientation-specific adaptation in human visual cortex. J Neurosci 23: 8781-8787, 2003.

Bradley A, Switkes E, and De Valois K. Orientation and spatial frequency selectivity of adaptation to color and luminance gratings. Vision Res 28: 841-856, 1988.

Brainard DH. The psychophysics toolbox. Spat Vis 10: 433-436, 1997.

Carandini M, Barlow HB, O'Keefe LP, Poirson AB, and Movshon JA. Adaptation to contingencies in macaque primary visual cortex. Philos Trans $R$ Soc Lond B 352: 1149-1154, 1997.

Carandini M, Movshon JA, and Ferster D. Pattern adaptation and crossorientation interactions in the primary visual cortex. Neuropharmacology 37: 501-511, 1998.

Chaudhuri A. Modulation of the motion aftereffect by selective attention. Nature 344: 60-62, 1990.

Chaudhuri A and Albright TD. Neuronal responses to edges defined by luminance vs. temporal texture in macaque area V1. Vis Neurosci 14: 949-962, 1997. 
Dakin SC and Mareschal I. Sensitivity to contrast modulation depends on carrier spatial frequency and orientation. Vision Res 40: 311-329, 2000.

De Valois KK. Spatial frequency adaptation can enhance contrast sensitivity. Vision Res 17: 1057-1065, 1977.

DeYoe EA, Carman GJ, Bandettini P, Glickman S, Wieser J, Cox R, Miller D, and Neitz J. Mapping striate and extrastriate visual areas in human cerebral cortex. Proc Natl Acad Sci USA 93: 2382-2386, 1996.

Dumoulin SO, Baker CLJr, Hess RF, and Evans AC. Cortical specialization for processing first- and second-order motion. Cereb Cortex 13: 1375-1385, 2003.

Dupont P, Sary G, Peuskens H, and Orban GA. Cerebral regions processing first- and higher-order motion in an opposed-direction discrimination task. Eur J Neurosci 17: 1509-1517, 2003.

Efron B and Tibshirani RJ. An Introduction to the Bootstrap. New York: Chapman and Hall, 1993.

Eger E, Henson RN, Driver J, and Dolan RJ. BOLD repetition decreases in object-responsive ventral visual areas depend on spatial attention. J Neurophysiol 92: 1241-1247, 2004.

Ellemberg D, Allen HA, and Hess RF. Investigating local network interactions underlying first- and second-order processing. Vision Res 44: 17871797, 2004.

Engel SA. Adaptation of oriented and unoriented color-selective neurons in human visual areas. Neuron 45: 613-623, 2005.

Engel SA, Rumelhart DE, Wandell BA, Lee AT, Glover GH, Chichilnisky EJ, and Shadlen MN. fMRI of human visual cortex. Nature 369: 525, 1994.

Felleman DJ and Van Essen DC. Distributed hierarchical processing in the primate cerebral cortex. Cereb Cortex 1: 1-47, 1991.

Festman $\mathbf{Y}$ and Ahissar M. Attentional states and the degree of visual adaptation to gratings. Neural Netw 17: 849-860, 2004.

Gallant JL, Braun J, and Van Essen DC. Selectivity for polar, hyperbolic, and Cartesian gratings in macaque visual cortex. Science 259: 100-103, 1993.

Gallant JL, Connor CE, Rakshit S, Lewis JW, and Van Essen DC. Neural responses to polar, hyperbolic, and Cartesian gratings in area V4 of the macaque monkey. J Neurophysiol 76: 2718-2739, 1996.

Ghose GM and Ts'o DY. Form processing modules in primate area V4. J Neurophysiol 77: 2191-2196, 1997.

Grill-Spector K, Kushnir T, Edelman S, Itzchak Y, and Malach R. Cue-invariant activation in object-related areas of the human occipital lobe. Neuron 21: 191-202, 1998a.

Grill-Spector K, Kushnir T, Hendler T, Edelman S, Itzchak Y, and Malach R. A sequence of object-processing stages revealed by fMRI in the human occipital lobe. Hum Brain Map 6: 316-328, 1998 b.

Grosof DH, Shapley RM, and Hawken MJ. Macaque V1 neurons can signal 'illusory' contours. Nature 365: 550-552, 1993.

Hammett ST and Snowden RJ. The effect of contrast adaptation on briefly presented stimuli. Vision Res 35: 1721-1725, 1995.

He S and MacLeod DI. Orientation-selective adaptation and tilt after-effect from invisible patterns. Nature 411: 473-476, 2001.

Hegde J and Van Essen DC. Selectivity for complex shapes in primate visual area V2. J Neurosci 20: RC61, 2000.

Hegde $\mathbf{J}$ and Van Essen DC. Strategies of shape representation in macaque visual area V2. Vis Neurosci 20: 313-328, 2003

Huk AC, Dougherty RF, and Heeger DJ. Retinotopy and functional subdivision of human areas MT and MST. J Neurosci 22: 7195-7205, 2002.

Huk AC and Heeger DJ. Pattern-motion responses in human visual cortex. Nat Neurosci 5: 72-75, 2002.

Huk AC, Ress D, and Heeger DJ. Neuronal basis of the motion aftereffect reconsidered. Neuron 32: 161-172, 2001.

Ito $\mathbf{M}$ and Komatsu $\mathbf{H}$. Representation of angles embedded within contour stimuli in area V2 of macaque monkeys. $J$ Neurosci 24: 3313-3324, 2004.

Jenkinson M, Bannister P, Brady M, and Smith S. Improved optimization for the robust and accurate linear registration and motion correction of brain images. Neuroimage 17: 825-841, 2002.

Kanal E, Borgstede JP, Barkovich AJ, Bell C, Bradley WG, Felmlee JP, Froelich JW, Kaminski EM, Keeler EK, Lester JW, Scoumis EA, Zaremba LA, and Zinninger MD. American College of Radiology White Paper on MR safety. AJR Am J Roentgenol 178: 1335-1347, 2002.

Kastner S, De Weerd P, and Ungerleider LG. Texture segregation in the human visual cortex: a functional MRI study. J Neurophysiol 83: 24532457,2000

Kingdom FA, Keeble D, and Moulden B. Sensitivity to orientation modulation in micropattern-based textures. Vision Res 35: 79-91, 1995.
Kingdom FA, Prins N, and Hayes A. Mechanism independence for texturemodulation detection is consistent with a filter-rectify-filter mechanism. Vis Neurosci 20: 65-76, 2003.

Kohn A and Movshon JA. Neuronal adaptation to visual motion in area MT of the macaque. Neuron 39: 681-691, 2003.

Kohn A and Movshon JA. Adaptation changes the direction tuning of macaque MT neurons. Nat Neurosci 7: 764-772, 2004.

Kourtzi $\mathbf{Z}$ and Kanwisher $\mathbf{N}$. Cortical regions involved in perceiving object shape. J Neurosci 20: 3310-3318, 2000.

Kourtzi $\mathbf{Z}$ and Kanwisher N. Representation of perceived object shape by the human lateral occipital complex. Science 293: 1506-1509, 2001.

Kourtzi Z, Tolias AS, Altmann CF, Augath M, and Logothetis NK. Integration of local features into global shapes: monkey and human FMRI studies. Neuron 37: 333-346, 2003.

Landy MS and Graham N. Visual perception of texture. In: The Visual Neurosciences, edited by Chalupa LM and Werner JS. Cambridge, MA: MIT Press, 2004, p. 1106-1118

Landy MS and Oruç I. Properties of second-order spatial frequency channels. Vision Res 42: 2311-2329, 2002.

Larsson J. Imaging Vision: Functional Mapping of Intermediate Visual Processes in Man (PhD thesis). Stockholm: Karolinska Institutet, 2001.

Larsson J, Landy MS, Schluppeck D, and Heeger DJ. Orientation-selective adaptation to first- and second-order stimuli in human visual cortex measured with FMRI. Soc Neurosci Abstr 911.917, 2003.

Ledgeway T and Smith AT. Evidence for separate motion-detecting mechanisms for first- and second-order motion in human vision. Vision Res 34: 2727-2740, 1994

Lerner Y, Hendler T, and Malach R. Object-completion effects in the human lateral occipital complex. Cereb Cortex 12: 163-177, 2002.

Leventhal AG, Wang Y, Schmolesky MT, and Zhou Y. Neural correlates of boundary perception. Vis Neurosci 15: 1107-1118, 1998.

Logothetis NK, Pauls J, Augath M, Trinath T, and Oeltermann A. Neurophysiological investigation of the basis of the fMRI signal. Nature 412: 150-157, 2001.

Lui LL, Bourne JA, and Rosa MG. Single-unit responses to kinetic stimuli in New World monkey area V2: physiological characteristics of cueinvariant neurones. Exp Brain Res 162: 100-108, 2005.

Mahon LE and De Valois RL. Cartesian and non-Cartesian responses in LGN, V1, and V2 cells. Vis Neurosci 18: 973-981, 2001.

Malach R, Reppas JB, Benson RR, Kwong KK, Jiang H, Kennedy WA, Ledden PJ, Brady TJ, Rosen BR, and Tootell RB. Object-related activity revealed by functional magnetic resonance imaging in human occipital cortex. Proc Natl Acad Sci USA 92: 8135-8139, 1995.

Marcar VL, Raiguel SE, Xiao D, and Orban GA. Processing of kinetically defined boundaries in areas V1 and V2 of the macaque monkey. J Neurophysiol 84: 2786-2798, 2000.

Mareschal I and Baker CL Jr. A cortical locus for the processing of contrast-defined contours. Nat Neurosci 1: 150-154, 1998a.

Mareschal I and Baker CL Jr. Temporal and spatial response to secondorder stimuli in cat area 18. J Neurophysiol 80: 2811-2823, 1998b.

Mareschal I and Baker CL Jr. Cortical processing of second-order motion. Vis Neurosci 16: 527-540, 1999.

Montaser-Kouhsari Land Rajimehr R. Attentional modulation of adaptation to illusory lines. J Vis 4: 434-444, 2004.

Moradi F, Koch C, and Shimojo S. Face adaptation depends on seeing the face. Neuron 45: 169-175, 2005.

Morgan MJ, Mason AJ, and Baldassi S. Are there separate first-order and second-order mechanisms for orientation discrimination? Vision Res 40: 1751-1763, 2000.

Neri P, Bridge H, and Heeger DJ. Stereoscopic processing of absolute and relative disparity in human visual cortex. J Neurophysiol 92: 1880-1891, 2004

Nestares $\mathrm{O}$ and Heeger DJ. Robust multiresolution alignment of MRI brain volumes. Magn Reson Med 43: 705-715, 2000.

Nishida S, Sasaki Y, Murakami I, Watanabe T, and Tootell RB. Neuroimaging of direction-selective mechanisms for second-order motion. $\mathrm{J} \mathrm{Neu}$ rophysiol 90: 3242-3254, 2003

Ogawa S, Lee TM, Kay AR, and Tank DW. Brain magnetic resonance imaging with contrast dependent on blood oxygenation. Proc Natl Acad Sci USA 87: 9868-9872, 1990.

O'Keefe LP and Movshon JA. Processing of first- and second-order motion signals by neurons in area MT of the macaque monkey. Vis Neurosci 15: $305-317,1998$ 
Olavarria JF, DeYoe EA, Knierim JJ, Fox JM, and van Essen DC. Neural responses to visual texture patterns in middle temporal area of the macaque monkey. J Neurophysiol 68: 164-181, 1992.

Pantle A and Sekuler R. Size-detecting mechanisms in human vision. Science 162: 1146-1148, 1968.

Pasupathy A and Connor CE. Responses to contour features in macaque area V4. J Neurophysiol 82: 2490-2502, 1999.

Pasupathy A and Connor CE. Shape representation in area V4: positionspecific tuning for boundary conformation. J Neurophysiol 86: 2505-2519, 2001.

Pelli DG. The VideoToolbox software for visual psychophysics: transforming numbers into movies. Spat Vis 10: 437-442, 1997.

Press WA, Brewer AA, Dougherty RF, Wade AR, and Wandell BA. Visual areas and spatial summation in human visual cortex. Vision Res 41: 13211332, 2001.

Purdon PL and Weisskoff RM. Effect of temporal autocorrelation due to physiological noise and stimulus paradigm on voxel-level false-positive rates in fMRI. Hum Brain Mapp 6: 239-249, 1998.

$\operatorname{Rezec}$ A, Krekelberg B, and Dobkins KR. Attention enhances adaptability: evidence from motion adaptation experiments. Vision Res 44: 3035-3044, 2004.

Rockland KS and Pandya DN. Laminar origins and terminations of cortical connections of the occipital lobe in the rhesus monkey. Brain Res 179: 3-20, 1979.

Rossi AF, Desimone R, and Ungerleider LG. Contextual modulation in primary visual cortex of macaques. J Neurosci 21: 1698-1709, 2001.

Salin PA and Bullier J. Corticocortical connections in the visual system: structure and function. Physiol Rev 75: 107-154, 1995.

Sary G, Vogels R, Kovacs G, and Orban GA. Responses of monkey inferior temporal neurons to luminance-, motion-, and texture-defined gratings. J Neurophysiol 73: 1341-1354, 1995.

Sary G, Vogels R, and Orban GA. Cue-invariant shape selectivity of macaque inferior temporal neurons. Science 260: 995-997, 1993.

Schluppeck D, Glimcher PW, and Heeger DJ. Topographic organization for delayed saccades in human posterior parietal cortex. J Neurophysiol 94: 1372-1384, 2005

Schofield AJ and Georgeson MA. Sensitivity to modulations of luminance and contrast in visual white noise: separate mechanisms with similar behaviour. Vision Res 39: 2697-2716, 1999.

Sclar G, Lennie P, and DePriest DD. Contrast adaptation in striate cortex of macaque. Vision Res 29: 747-755, 1989.

Scott-Samuel NE and Georgeson MA. Does early non-linearity account for second-order motion? Vision Res 39: 2853-2865, 1999.

Seiffert AE, Somers DC, Dale AM, and Tootell RB. Functional MRI studies of human visual motion perception: texture, luminance, attention and aftereffects. Cereb Cortex 13: 340-349, 2003.

Sereno MI, Dale AM, Reppas JB, Kwong KK, Belliveau JW, Brady TJ, Rosen BR, and Tootell RB. Borders of multiple visual areas in humans revealed by functional magnetic resonance imaging. Science 268: 889-893, 1995.

Shmuel A, Yacoub E, Pfeuffer J, Van de Moortele PF, Adriany G, Hu X, and Ugurbil K. Sustained negative BOLD, blood flow and oxygen consumption response and its coupling to the positive response in the human brain. Neuron 36: 1195-1210, 2002.

Silver MA, Ress D, and Heeger DJ. Topographic maps of visual spatial attention in human parietal cortex. J Neurophysiol 94: 1358-1371, 2005.

Smith AM, Lewis BK, Ruttimann UE, Ye FQ, Sinnwell TM, Yang Y, Duyn JH, and Frank JA. Investigation of low frequency drift in fMRI signal. Neuroimage 9: 526-533, 1999.

Smith AT, Greenlee MW, Singh KD, Kraemer FM, and Hennig J. The processing of first- and second-order motion in human visual cortex assessed by functional magnetic resonance imaging (fMRI). J Neurosci 18: 38163830,1998

Snowden RJ and Hammett ST. Spatial frequency adaptation: threshold elevation and perceived contrast. Vision Res 36: 1797-1809, 1996.
Sohn W, Papathomas TV, Blaser E, and Vidnyanszky Z. Object-based cross-feature attentional modulation from color to motion. Vision Res 44: 1437-1443, 2004.

Solomon SG, Peirce JW, Dhruv NT, and Lennie P. Profound contrast adaptation early in the visual pathway. Neuron 42: 155-162, 2004.

Spivey MJ and Spirn MJ. Selective visual attention modulates the direct tilt aftereffect. Percept Psychophys 62: 1525-1533, 2000.

Sutter A, Sperling G, and Chubb C. Measuring the spatial frequency selectivity of second-order texture mechanisms. Vision Res 35: 915-924, 1995.

Thomas OM, Cumming BG, and Parker AJ. A specialization for relative disparity in V2. Nat Neurosci 5: 472-478, 2002.

Tolias AS, Keliris GA, Smirnakis SM, and Logothetis NK. Neurons in macaque area V4 acquire directional tuning after adaptation to motion stimuli. Nat Neurosci 8: 591-593, 2005.

Tootell RB, Hadjikhani N, Hall EK, Marrett S, Vanduffel W, Vaughan JT, and Dale AM. The retinotopy of visual spatial attention. Neuron 21: 1409-1422, 1998a.

Tootell RB, Hadjikhani NK, Vanduffel W, Liu AK, Mendola JD, Sereno MI, and Dale AM. Functional analysis of primary visual cortex (V1) in humans. Proc Natl Acad Sci USA 95: 811-817, 1998b.

Tootell RB, Mendola JD, Hadjikhani NK, Ledden PJ, Liu AK, Reppas JB, Sereno MI, and Dale AM. Functional analysis of V3A and related areas in human visual cortex. J Neurosci 17: 7060-7078, 1997.

Tootell RB, Reppas JB, Kwong KK, Malach R, Born RT, Brady TJ, Rosen BR, and Belliveau JW. Functional analysis of human MT and related visual cortical areas using magnetic resonance imaging. J Neurosci 15: 3215-3230, 1995.

von der Heydt $\mathbf{R}$ and Peterhans E. Mechanisms of contour perception in monkey visual cortex. I. Lines of pattern discontinuity. J Neurosci 9: 1731-1748, 1989

von der Heydt R, Peterhans E, and Baumgartner G. Illusory contours and cortical neuron responses. Science 224: 1260-1262, 1984.

Wade AR, Brewer AA, Rieger JW, and Wandell BA. Functional measurements of human ventral occipital cortex: retinotopy and colour. Philos Trans $R$ Soc Lond B 357: 963-973, 2002.

Wandell BA, Brewer AA, and Dougherty RF. Visual field map clusters in human cortex. Philos Trans R Soc Lond B 360: 693-707, 2005.

Watson JD, Myers R, Frackowiak RS, Hajnal JV, Woods RP, Mazziotta JC, Shipp S, and Zeki S. Area V5 of the human brain: evidence from a combined study using positron emission tomography and magnetic resonance imaging. Cereb Cortex 3: 79-94, 1993.

Wenderoth P, Watson JD, Egan GF, Tochon-Danguy HJ, and O'Keefe GJ. Second order components of moving plaids activate extrastriate cortex: a positron emission tomography study. Neuroimage 9: 227-234, 1999.

Wichmann FA and Hill NJ. The psychometric function. I. Fitting, sampling, and goodness of fit. Percept Psychophys 63: 1293-1313, 2001a

Wichmann FA and Hill NJ. The psychometric function. II. Bootstrap-based confidence intervals and sampling. Percept Psychophys 63: 1314-1329, 2001b.

Yi DJ and Chun MM. Attentional modulation of learning-related repetition attenuation effects in human parahippocampal cortex. J Neurosci 25: 3593 3600, 2005

Zarahn E, Aguirre GK, and D'Esposito M. Empirical analyses of BOLD fMRI statistics. I. Spatially unsmoothed data collected under null-hypothesis conditions. Neuroimage 5: 179-197, 1997.

Zenger-Landolt Band Heeger DJ. Response suppression in V1 agrees with psychophysics of surround masking. J Neurosci 23: 6884-6893, 2003.

Zhou YX and Baker CL Jr. A processing stream in mammalian visual cortex neurons for non-Fourier responses. Science 261: 98-101, 1993.

Zhou YX and Baker CL Jr. Envelope-responsive neurons in areas 17 and 18 of cat. J Neurophysiol 72: 2134-2150, 1994.

Zhou YX and Baker CL Jr. Spatial properties of envelope-responsive cells in area 17 and 18 neurons of the cat. J Neurophysiol 75: 1038-1050, 1996. 